\title{
Modeling Eclipses in the Classical Nova V Persei: The Role of the Accretion Disk Rim
}

\author{
A. W. Shafter \\ Astronomy Department, San Diego State University, San Diego, CA 92182 \\ aws@nova.sdsu.edu \\ and \\ K. A. Misselt \\ Steward Observatory, Tucson, AZ 85719 \\ misselt@as.arizona.edu
}

\begin{abstract}
Multicolor $(B V R I)$ light curves of the eclipsing classical nova V Per are presented, and a total of twelve new eclipse timings are measured for the system. When combined with previous eclipse timings from the literature, these timings yield a revised ephemeris for the times of mid-eclipse given by $\mathrm{JD}_{\odot}=2,447,442.8260(1)+0.107123474(3) E$. The eclipse profiles are analyzed with a parameter-fitting model that assumes four sources of luminosity: a white dwarf primary star, a main-sequence secondary star, a flared accretion disk with a rim, and a bright spot at the intersection of the mass-transfer stream and the disk periphery. Model parameters include the temperatures of the white dwarf $\left(T_{1}\right)$ and the secondary star $\left(T_{2}\right)$, the radius $\left(R_{\mathrm{d}}\right)$ and temperature $\left(T_{\mathrm{d}}\right)$, of the disk periphery, the inner disk radius $\left(R_{\text {in }}\right)$, the disk power-law temperature exponent $(\alpha)$ and thickness $\left(h_{\mathrm{r}}\right)$, and a bright spot temperature enhancement factor $\left(\chi_{\mathrm{s}}\right)$. A matrix of model solutions are computed, covering an extensive range of plausible parameter values. The solution matrix is then explored to determine the optimum values for the fitting parameters and their associated errors. For models that treat the accretion disk as a flat structure without a rim, optimum fits require that the disk have a flat temperature profile. Although models with a truncated inner disk $\left(R_{\text {in }}>>R_{1}\right)$ result in a steeper temperature profile, steadystate models with a temperature profile characterized by $T(r) \propto r^{-3 / 4}$ are found only for models with a significant disk rim. A comparison of the observed brightness and color at mid-eclipse with the photometric properties of the best-fitting model suggests that $\mathrm{V}$ Per lies at a distance of $\sim 1 \mathrm{kpc}$.
\end{abstract}


Subject headings: binaries: eclipsing - novae, cataclysmic variables - stars: dwarf novae - stars: individual (V Persei)

\section{Introduction}

V Per is an 18th magnitude classical nova system (Nova Persei 1887), which reached an estimated magnitude of $m \sim 4-5$ at the peak of its eruption (see McLaughlin 1946). More than a century later, the system was discovered to be eclipsing by Shafter \& Abbott (1989) who established an orbital period of $2.57 \mathrm{hr}$. V Per is of particular interest because its orbital period places the system near the middle of the 2-3 hr "gap" in the orbital period distribution of cataclysmic variable stars (Kolb 1996; Ritter 1996). In addition to the possibility that V Per could have been formed in the period gap, Shafter \& Abbott discussed possible evolutionary scenarios that could have brought V Per to its current period under the assumption that the system evolved from an initially longer orbital period.

Eclipsing cataclysmic variables are valuable because they provide the opportunity to study the the radiative properties of accretion disks through analysis of the eclipse profiles. Generally, properties of the system are determined either through parameter-fitting models or through maximum-entropy eclipse mapping techniques (e.g. Horne 1985). In the former case, properties of the accretion disk, such as its radial temperature profile, must be specified in analytic form, while the latter method allows for an arbitrary disk emissivity distribution, and selects the "simplest" (maximum entropy) disk emissivity map.

Shortly after V Per was discovered to be eclipsing, Wood et al. (1992) analyzed the original monochromatic photometric data from Shafter \& Abbott (1989) using the maximum entropy eclipse-mapping technique. The principal result of their study was that the accretion disk in V Per had a relatively "flat" radial temperature profile that was considerably less steep than the $T(r) \propto r^{-3 / 4}$ profile predicted for a steady-state disk (e.g. Shakura \& Sunyaev 1973). Despite the inconsistency with the expected steady-state relation, the flat temperature profile seen in V Per is not unusual. Several eclipse mapping studies of novalike variables and dwarf novae in eruption - systems expected to harbor steady-state disks - have also exhibited surprisingly flat temperature profiles (e.g. Rutten et al. 1992; Robinson et al. 1999; Bíró 2000). This discrepancy has been explained by Smak (1994) who used an eclipse-mapping technique to analyze model eclipse profiles from disks with and without rims, demonstrating that the spurious temperature profiles found in previous studies of high inclination systems

$\left(z 80^{\circ}\right)$ were a result of a failure to model the disk rim. More recently, Knigge et al. (2000) used synthetic eclipse profiles of a rimmed disk from their study of DW UMa to show that self-occultation of the disk by its rim, in addition to the contribution of the rim to the total 
light, causes the eclipse profiles to be more $\mathbf{V}$-shaped than in the case of a flat disk. They argued following Smak (1994), that if these light curves were then modeled assuming the disk is flat (as in eclipse mapping), an erroneously flat temperature gradient would result. In the case of V Per, Wood et al. (1992) found that a model with a truncated inner disk, such as what one might expect if the white dwarf primary were strongly magnetic, had a temperature profile that was considerably steeper, similar to that expected for a steady-state disk.

Table 1 summarizes the properties of the 15 cataclysmic variables with measured periods between $2.25 \mathrm{hr}$ and $2.75 \mathrm{hr}$. Two of the stars are classified as classical novae (one of which is V Per), eight as magnetic systems (i.e. DQ Her or AM Her stars), two as novalike variables, two as SU UMa dwarf novae, and one as both a nova and a DQ Her star. Given that eight out of the 15 systems near the middle of the period gap, are in fact magnetic (i.e. either DQ Her or AM Her systems), it would not be surprising if Wood et al. (1992) were correct, and V Per turned out to be a magnetic system as well. In order to pursue this question further, and to explore how the addition of a disk rim would affect the model results, we have obtained multicolor eclipse light curves of $\mathrm{V}$ Per and analyzed the data with a new parameter-fitting eclipse model. In this paper, we present the results of that study.

\section{Observations}

Observations of V Per were carried out in 2002 October and December using the $2048^{2}$ CCD imager on the Steward Observatory Bok $2.3 \mathrm{~m}$ reflector. A summary of observations is presented in Table 2. To increase the time sampling efficiency, only a small subsection of the full array was read out. The subsection was centered on V Per and included several nearby stars that were used as comparison objects for differential photometry. Typical time resolutions were in the range of 40-60 sec.

The data were processed in a standard fashion (bias subtraction and flat-fielding) using IRAF. ${ }^{1}$ The individual images were subsequently aligned to a common coordinate system and aperture photometry was performed through a $6^{\prime \prime}$ aperture on V Per and two nearby comparison stars, one much brighter than V Per and the other of approximately equal brightness. Differential light curves of V Per were constructed with respect to the brighter comparison star and the stability of the atmospheric conditions was monitored using the

\footnotetext{
${ }^{1}$ IRAF (Image Reduction and Analysis Facility) is distributed by the National Optical Astronomy Observatories, which are operated by AURA, Inc., under cooperative agreement with the National Science Foundation.
} 
star of comparable brightness. In all cases, the monitor star showed no long-term drift, and had a point-to-point scatter of less than 0.02 differential magnitudes.

To calibrate our observations, several standard stars were observed on each night. Zero points were determined on each night using mean extinction values appropriate to the Kitt Peak site. On several nights, standards were observed over a sufficient range of airmass for a full solution including the extinction coefficient in each band. In all cases, we found extinction coefficients consistent with the mean values. All subsequently reported magnitudes were computed using nightly determined zero points and the mean extinction. The two comparison stars in the V Per field were calibrated by calibrating every individual frame of our observations on each night and computing the mean. The comparison star used to calibrate the V Per light curves is located at $\mathrm{RA}=2: 01: 54.3, \mathrm{DEC}=+56: 44: 38.7(2000.0)\left(\sim 35^{\prime \prime}\right.$ north of V Per), and is characterized by $B=16.90 \pm 0.03, V=16.11 \pm 0.03, R=15.60 \pm 0.03$, and $I=15.09 \pm 0.03$. The twelve calibrated eclipse light curves - three each in $B, V, R$, and $I$ - are shown in Figure 1. V Per is characterized by an out-of-eclipse magnitude of $V \simeq 18.1 \pm 0.1$ and $B-V \simeq 0.6 \pm 0.2$. The luminosity of the system appears to be relatively stable over the $\sim 2$ month time span covered by the observations, with the exception of the data obtained on 29 Oct 2002, when V Per appeared to be $\sim 0.4$ mag fainter than usual. Mean magnitudes and colors of V Per, both in and out of eclipse, are given in Table 3.

\section{Updated Ephemeris}

Eclipse timings for $\mathrm{V}$ Per extend back more than 15 years to the original eclipses discovered in the work of Shafter \& Abbott (1988). Table 4 summarizes all available timings of mid-eclipse from Shafter \& Abbott (1989), Dahm (1997), Katysheva et al. (2002), and from the present study. Following earlier studies, times of mid-eclipse for the twelve eclipses in the present study have been determined by fitting a parabola to the lower half of the eclipse data. A linear least-squares fit of the mid-eclipse times from Table 4 yields the following ephemeris for V Per:

$$
T_{\text {mid-eclipse }}=\mathrm{JD}_{\odot} 2,447,442.8260(1)+0.107123474(3) E .
$$

Residuals of the individual eclipse timings with respect to this ephemeris are also given

in Table 4, and are plotted as a function of cycle number in Figure 2. The period of V Per appears to be remarkably stable, with no evidence for any significant period change over the $\sim 15$ years that the eclipses of V Per have been monitored. 


\section{Eclipse Model}

The V Per data have been analyzed using an improved version of the eclipse modeling program described in Shafter et al. (2000, Paper I), and in Shafter \& Holland (2003, Paper II). The model considers four principal sources of light from the system: the white dwarf, the secondary star, the accretion disk, and the bright spot where the inter-star mass transfer stream impacts the periphery of the disk. The model light curve flux at a given orbital phase is computed by summing the contribution from the secondary star and the unocculted regions of the remaining three sources of radiation. The fluxes from all four sources of radiation have been corrected via the use of a linear limb-darkening law with coefficients, $\mu_{\lambda}$, given in van Hamme (1993). The program operates on the normalized light curve intensities, so in effect, we fit only the shapes and depths of the eclipse profiles. The program does, however, compute a model $B-V$ color from the assumed blackbody fluxes using the transformations given in Matthews \& Sandage (1963), which can then be compared with the observed $B-V$ color.

The major improvement in our model is in the treatment of the accretion disk, which is no longer confined to the orbital plane. The disk thickness perpendicular to the orbital plane now varies linearly with radius, resulting in a rim at the disk periphery. The flaring angle of the disk and the rim (half) height are parameterized by a fitting parameter, $h_{\mathrm{r}}=h / R_{\mathrm{d}}$, where $h$ is the disk height at its edge perpendicular to the orbital plane, and $R_{\mathrm{d}}$ is the disk radius. As before, the disk is assumed to radiate like an optically-thick blackbody, with a temperature distribution that follows a radial power-law profile given by:

$$
T(r)=T_{\mathrm{d}}\left(\frac{R_{\mathrm{d}}}{r}\right)^{\alpha},
$$

where $T_{\mathrm{d}}$ is the temperature of the rim at the outer edge of the disk. For an optically thick, steady-state disk, a characteristic value of $\alpha=0.75$ is expected (Shakura \& Sunyaev 1973).

Another improvement to earlier versions of our model involves the treatment of radiation from the secondary star. In the current model, the tidally-distorted shape of the Rochelobe-filling secondary star is fully taken into account, not only when computing the occulted regions of the white dwarf, disk, and bright spot, as in earlier versions of the code, but also when computing the contribution of the secondary star to the model flux. The flux from the white dwarf component is computed assuming it to be a spherical blackbody of temperature $T_{1}$. The radii of the white dwarf and secondary stars are computed from the orbital period and mass ratio as described in Paper I.

One of the significant advantages of the inclusion of a disk rim is that the bright spot can be more easily modeled. The presence of the disk rim provides both an isotropic and 
an anisotropic component to the bright spot radiation, so there is no need to parameterize these components separately as in Paper II. The isotropic component of the bright spot is produced as in Paper I, by radiation from a region of the disk's surface defined by the intersection of a circular area of radius $0.2 R_{\mathrm{d}}$ (centered on the point of intersection of the mass-transfer stream and the disk perimeter) and the accretion disk. The anisotropic component is produced by radiation from the corresponding azimuthal region of the disk rim. As before, the bright spot is assumed to radiate like a blackbody, the bright spot temperature parameterized by a multiplicative factor, $\chi_{\mathrm{s}}$, applied to the local disk temperature in the bright spot region.

For a given mass ratio and orbital inclination, the geometry of the eclipse is determined by dividing the primary's Roche lobe into a matrix of cells having Cartesian coordinates $(x, y, z)$, and then computing an occultation kernel $O(x, y, x, \phi)$ for eclipse by the lobe-filling secondary star. The $x$ and $y$ axes lie in the orbital plane with the positive $x$ axis pointing toward the secondary star, and the positive $y$ axis oriented $90^{\circ}$ counterclockwise as seen from the direction of the positive $z$ axis. For a given orbital phase, $\phi$, the occultation kernel takes on values of zero or one, depending on whether or not the cell is eclipsed. The monochromatic fluxes from the accretion disk at orbital phase $\phi$ are then given by the following summations. For the disk surface and disk rim, respectively, we have:

$$
\begin{gathered}
f_{\lambda}^{\mathrm{s}}(\phi)=\frac{\cos i}{d^{2}} \sum_{x=-R_{\mathrm{d}}}^{x=R_{\mathrm{d}}} \sum_{y=-\sqrt{R_{\mathrm{d}}^{2}-x^{2}}}^{y=\sqrt{R_{\mathrm{d}}^{2}-x^{2}}} I_{\lambda}^{\mathrm{s}}(x, y) \Delta x \Delta y O\left(x, y, z=h_{\mathrm{r}} \sqrt{x^{2}+y^{2}}, \phi\right), \\
f_{\lambda}^{\mathrm{r}}(\phi)=\frac{\sin i}{d^{2}} \sum_{\phi^{\prime}=-\pi / 2}^{\phi^{\prime}=\pi / 2} \sum_{z=-h_{\mathrm{r}} R_{\mathrm{d}}}^{z=h_{\mathrm{d}} R_{\mathrm{d}}} I_{\lambda}^{\mathrm{r}}(x, y)\left(R_{\mathrm{d}} \Delta \phi^{\prime}\right) \cos \phi^{\prime} \Delta z O(x, y, z, \phi),
\end{gathered}
$$

where $d$ is the distance to the system, $i$ is the orbital inclination, and $\phi^{\prime}=\phi+\tan ^{-1}(y / x)$ is the phase-modified disk azimuth angle. The Cartesian coordinates of the disk rim are given by $x=R_{\mathrm{d}} \cos \left(\phi^{\prime}-\phi\right)$ and $y=R_{\mathrm{d}} \sin \left(\phi^{\prime}-\phi\right)$.

The disk surface and rim are assumed to radiate as blackbodies, with limb-darkened specific intensities given by:

$$
\begin{gathered}
I_{\lambda}^{\mathrm{s}}(x, y)=B_{\lambda}\left(T_{\mathrm{d}} S_{\mathrm{x}, \mathrm{y}}\left[R_{\mathrm{d}} / \sqrt{x^{2}+y^{2}}\right]^{\alpha}\right)\left(1-\mu_{\lambda}+\mu_{\lambda} \cos \gamma\right), \\
I_{\lambda}^{\mathrm{r}}(x, y)=B_{\lambda}\left(T_{\mathrm{d}} S_{\mathrm{x}, \mathrm{y}}\right)\left(1-\mu_{\lambda}+\mu_{\lambda} \sin i \cos \phi^{\prime}\right),
\end{gathered}
$$

where $\cos \gamma=\cos \beta \cos i-\sin i \sin \beta \cos \phi^{\prime}$ is the cosine of the angle between the line-ofsight and the normal to the disk surface, $\beta=\tan ^{-1}\left(h_{\mathrm{r}}\right)$ is the half-angle of the disk flare, and $\mu_{\lambda}$ are the linear limb-darkening coefficients. The parameter $S_{\mathrm{x}, \mathrm{y}}$ modifies the outer 
disk temperature in the vicinity of the intersection of the mass transfer stream and the disk periphery $\left(x_{\mathrm{s}}, y_{\mathrm{s}}\right)$ by the factor $\chi_{\mathrm{s}}$ as follows:

$$
S_{\mathrm{x}, \mathrm{y}}=\left\{\begin{array}{ccc}
\chi_{\mathrm{s}} & \text { when } & \sqrt{\left(x-x_{\mathrm{s}}\right)^{2}+\left(y-y_{\mathrm{s}}\right)^{2}}<R_{\mathrm{spot}} \\
1 & \text { otherwise. }
\end{array}\right.
$$

The fluxes for the secondary star and the white dwarf primary are computed as follows. For the secondary star we have:

$$
f_{\lambda}^{2}(\phi)=\frac{1}{d^{2}} \sum B_{\lambda}\left(T_{2}\right)\left[1-\mu_{\lambda}+\mu_{\lambda} \cos \xi(\phi)\right] \cos \xi(\phi) \Delta A,
$$

where $\Delta A$ is an element of area on the surface of the tidally-distorted secondary star with a normal vector inclined at an angle $\xi$ to our line of sight. The white dwarf primary is assumed to be a spherical blackbody of temperature $T_{1}$ and radius $R_{1}$. Its integrated flux is given by:

$$
f_{\lambda}^{1}(\phi)=\frac{1}{d^{2}} B_{\lambda}\left(T_{1}\right)\left(1-\mu_{\lambda} / 3\right) Q\left(R_{\text {in }}\right) \pi R_{1}^{2} O(0,0,0, \phi)
$$

where the parameter $Q$ applies a correction depending on whether the lower hemisphere of the white dwarf is shadowed by the inner edge of the disk, $R_{\text {in }}$, as follows:

$$
Q\left(R_{\text {in }}\right)=\left\{\begin{array}{ccc}
\frac{1+\cos i}{2} & \text { when } & R_{\text {in }}=R_{1} \\
1 & \text { when } & R_{\text {in }}>R_{1}
\end{array}\right.
$$

Finally, the normalized light curves, independent of the distance to the system, are given by:

$$
l_{\lambda}(\phi)=\frac{f_{\lambda}^{\mathrm{s}}(\phi)+f_{\lambda}^{\mathrm{r}}(\phi)+f_{\lambda}^{1}(\phi)+f_{\lambda}^{2}(\phi)}{f_{\lambda}^{\mathrm{s}}\left(\phi_{\mathrm{n}}\right)+f_{\lambda}^{\mathrm{r}}\left(\phi_{\mathrm{n}}\right)+f_{\lambda}^{1}\left(\phi_{\mathrm{n}}\right)+f_{\lambda}^{2}\left(\phi_{\mathrm{n}}\right)},
$$

where a normalization phase of $\phi_{\mathrm{n}}=0.2$ has been adopted to represent the out-of-eclipse light level.

\subsection{Input Parameters}

The input parameters required by the model are summarized in Table 5. They can be divided into two general categories: fixed parameters and fitting parameters. The fixed parameters are those that are either known, a priori, such as the orbital period, or parameters whose values can be estimated using assumptions implicit in the model. The fitting parameters, on the other hand, cannot be specified initially, and are varied during the fitting procedure. 
Since the orbital period and eclipse width are known, the masses and dimensions of the binary system can be computed once the mass ratio is specified (see Paper I). Unfortunately, the mass ratio of V Per has not been directly measured; however, its value can be estimated as follows. A lower limit on the mass ratio is established by noting that the observed eclipse width, $\Delta \phi=0.073$ (Wood et al. 1992), requires that $q\left(=M_{2} / M_{1}\right) \gtrsim 0.2$ for orbital inclinations, $i<90^{\circ}$ (Chanan et al. 1976). In addition, the requirement for stable mass transfer can be used to place an upper limit on the mass ratio given the mass of the secondary star. The dependence of $M_{2}$ on $q$ is weak, and to first order the mass of the secondary star can be expressed as a function of the orbital period alone. From Warner (1995, eqn. 2.100) we can write $\left(M_{2} / M_{\odot}\right) \simeq 0.065 P^{5 / 4}(h r)$. For an orbital period of $2.57 \mathrm{hr}$, we can estimate $M_{2} \simeq 0.2 M_{\odot}$. For secondary star masses less than $0.4 M_{\odot}$, stable mass transfer requires $q \lesssim 2 / 3$ (e.g. Politano 1988). Thus, the mass ratio of $\mathrm{V}$ Per must lie roughly in the range $0.2 \lesssim q \lesssim 0.6$. Since for nova systems the mass of the white dwarf is almost certainly greater than $0.5 M_{\odot}$, we can further reduce the upper limit of plausible mass ratios to $q \simeq 0.4$, with the most likely value lying closer to $q \simeq 0.2$, given that the average white dwarf masses in classical nova systems is believed to be of order $0.85 M_{\odot}$ (Smith \& Dhillon 1998). In order to explore the effect of the mass ratio on our analysis, we will explore solutions for mass ratios of both $q=0.2$ and $q=0.4$, with the former representing the most likely model. Following the analysis described in Paper I, the masses and dimensions of the V Per system have been computed for both of our representative mass ratios, and are summarized in Table 6 .

In addition to the mass ratio, the spectral type and temperature of the secondary star can be estimated prior to beginning the fitting procedure. In their statistical study of the properties of cataclysmic variable stars, Smith \& Dhillon (1998) found an empirical relation between the orbital period and spectral type of the secondary star. For an orbital period of $2.57 \mathrm{hr}$, the most likely spectral type is between M4V and M5V. According to Popper (1980) a main sequence star with a spectral type in this range is expected to have a temperature of $\sim 3300 \mathrm{~K}$. Thus, for the purposes of our study, we will adopt $T_{2}=3300 \mathrm{~K}$, and not vary this parameter during the fitting procedure.

Another input parameter than can be constrained to a limited extent is the disk rim thickness, $h_{\mathrm{r}}=h / R_{\mathrm{d}}$, where $h$ is the height of the rim perpendicular to the plane of the disk and $R_{\mathrm{d}}$ is the disk radius. According to the accretion disk models of Cannizzo (2001) the pressure scale height near the disk midplane gives $h_{\mathrm{r}} \simeq 0.01$ to a good approximation. Unfortunately, this theoretical scale height, most often referred to by disk modelers, is distinct from aspect ratios, $h / r \simeq 0.03-0.1$, that are measured by eclipse mapping techniques, and relevant to our study. In particular, Cannizzo \& Wheeler (1984) carried out vertical integrations of accretion disk structure and showed that, even under standard $\alpha$-disk theory, the disk photosphere can lie at $\sim 3-4$ pressure scale heights. According to Warner (1995, 
eqn. 2.52), similar studies by Meyer \& Meyer-Hofmeister (1982) and Smak (1992) show that the rim height can be approximated by $h_{\mathrm{r}} \simeq 0.038 \dot{M}_{16}^{3 / 20}$. For plausible mass accretion rates between $\sim 10^{16} \mathrm{~g} \mathrm{~s}^{-1}$ and $\sim 10^{18} \mathrm{~g} \mathrm{~s}^{-1}$, we estimate rim heights of in the range of $h_{\mathrm{r}} \sim 0.04$ to $h_{\mathrm{r}} \sim 0.08$, which is consistent with our earlier estimate. To cover the range, we consider specific rim heights of both $h_{\mathrm{r}}=0.04$ and $h_{\mathrm{r}}=0.08$ in our models of V Per. For comparisons we have also considered a $h_{\mathrm{r}}=0.0$ "flat disk" case for comparison with previous studies. The values of the remaining parameters, the "fitting parameters", which include the radial disk temperature parameter $\alpha$, and the temperatures of the disk rim, bright spot, and component stars, are constrained through the model fit.

\section{Light-Curve Fitting}

To begin the fitting procedure, we select plausible ranges of values for the five fitting parameters, indicated in Table 5 as "variable". Then each parameter is varied one at a time through a suitable range while the remaining parameters are held constant. In this way a 5-dimensional parameter space of models is explored for plausible solutions. The goodness of fit at each stage is determined using a standard $\chi^{2}$ test. The deviations between the model and the data are determined for each of the four colors, and the final $\chi^{2}$ statistic is determined by weighting each of the colors equally. Since we model the light curve near eclipse only, the calculation of $\chi^{2}$ has been restricted to orbital phases between $\phi=-0.12$ and $\phi=0.12$, which corresponds roughly to the time just prior to the onset of disk ingress to the completion of bright-spot egress. The range over which each parameter is varied has been chosen to be sufficiently large so that all plausible model solutions were explored. A thorough sampling required eight values to be considered for each fitting parameter. Thus, a total of $32,768\left(=8^{5}\right)$ models were computed for each mass ratio and rim thickness considered. The best-fitting solutions for our grid of models are given Table 7.

In their earlier monochromatic ("white light") eclipse-mapping study of V Per, Wood et al. (1992) found that the brightness temperature gradient of the accretion disk could be made consistent with a steady-state $(\alpha=0.75)$ temperature profile only if the inner edge of the accretion disk was of order $R_{\text {in }} \sim 0.2 R_{\mathrm{L} 1}$, where $R_{\mathrm{L} 1}$ is the distance from the center of the white dwarf to the inner Lagrangian point. These authors pointed out that such a situation might arise if the inner accretion disk was disrupted by the white dwarf's magnetic field, as is believed to be the case in intermediate polar systems (e.g. Warner 1995). To explore this possibility further, we have computed an additional set of eclipse models where the inner disk radius has been set at the value, $R_{\text {in }}=0.2 R_{\mathrm{L} 1}$, adopted by Wood et al. (1992). The results of our intermediate polar models are shown in parentheses in Table 7. 


\subsection{The Model Solution Distributions}

Exploring the matrix of possible solutions reveals that many combinations of parameters produce good fits to the data. The goal is to determine which particular combination of parameters best represents the true model for $\mathrm{V}$ Per. The best model fits are characterized by a reduced chi-square, $\chi_{\nu}^{2} \simeq 1.5$; however, a wide range of parameters will give comparable fits to the light curves. To explore the range of plausible solutions, frequency distributions for the top 100 best-fitting model solutions were constructed for each of the fitting parameters. Figures 3-5 show the frequency distributions of the five fitting parameters, together with the the model $B-V$ color, for our preferred mass ratio of $q=0.20$. Figure 3 shows the distributions for our rimless model with $h_{\mathrm{r}}=0.0$, with Figures 4 and 5 showing the distributions for $h_{\mathrm{r}}=0.04$, and $h_{\mathrm{r}}=0.08$, respectively. The mean and standard deviation of each parameter distribution, along with the mean $\chi_{\nu}^{2}$ for each model, are summarized in Tables 8 and 9 for the standard and truncated inner disk models, respectively. The mean $\chi_{\nu}^{2}$ of the top 100 model solutions is significantly higher in the rimless disk models, but still reflects acceptable fits to the data. As long as the number of model solutions included in the distributions is sufficiently small so that $\left\langle\chi_{\nu}^{2}\right\rangle \lesssim 1.8$ (which represents a plausible fit to the data), the distribution means do not depend strongly on the precise number of model solutions included in the distributions.

The frequency distributions reveal how well a given parameter is constrained by the model fit. If a parameter is tightly constrained by the data, its frequency distribution will be narrow and centered on its optimum value. Thus, the mean and standard deviation of each distribution not only provide an estimate of the optimum value for a specific parameter, but also a quantitative assessment of its uncertainty. The most tightly constrained parameter is clearly the disk radius, $R_{\mathrm{d}}$, where a radius of $\sim 70-75 \%$ of the distance to the inner Lagrangian point is required by virtually all the models. The value of this parameter is primarily determined by the phase width where the disk eclipse begins and ends, and is relatively insensitive to other system parameters (e.g. Sulkanen et al. 1981). The light curves do not exhibit any significant asymmetry of the eclipse profile, and there is no prominent "hump" prior to eclipse, so it is not surprising that none of the models considered required significant radiation from the bright spot in the system. Furthermore, the eclipse profile, which lacks the sharp transitions on ingress and egress characteristic of the white dwarf eclipse suggest that the white dwarf star does not contribute significantly to the system light. Thus, as expected, none of the models we considered required a luminous (hot) white dwarf. As an example, Figure 6 shows the best-fitting $q=0.20$ and $h_{\mathrm{r}}=0.08$ model plotted together with the phased eclipse data. The best fitting models for $q=0.4$, and for other rim thicknesses fit the data almost as well, and cannot be ruled out based on the fit alone. 


\section{Discussion}

\subsection{Disk Structure}

Monochromatic eclipses of V Per have been studied by Wood et al. (1992) using a maximum entropy eclipse-mapping technique. A principal conclusion of their study was that the brightness temperature distribution of the accretion disk was flatter than that expected for a steady-state disk. These authors showed, however, that if the inner disk in $\mathrm{V}$ Per were disrupted out to $R_{\text {in }} \sim 0.15-0.25 R_{\mathrm{L} 1}$, as might be expected if $\mathrm{V}$ Per harbors a magnetic white dwarf, then a brightness temperature gradient very close to that expected for a steady-state disk could be achieved.

Our multi-color eclipse simulations are qualitatively in agreement with the results of Wood et al. (1992). Referring to Table 7, we see that for a given mass ratio and rim thickness, the intermediate polar models are generally characterized by slightly larger values of the disk temperature parameter, $\alpha$. However, in our case, only models with a disk rim produced a best-fitting value of $\alpha$ close to the steady-state value. Only two cases - the $q=0.2$, $h_{\mathrm{r}}=0.08$ standard model and the $q=0.4, h_{\mathrm{r}}=0.08$ intermediate polar model - resulted in a value of $\alpha=0.75$. It is noteworthy that for a given rim height the value of $\alpha$ is systematically higher in all of the intermediate polar models compared with the standard models, underscoring the synergistic effect between the rim and the truncated inner disk in achieving a steady-state disk solution. For the orbital inclination of $85.4^{\circ}$ required by our $q=0.2$ model, the central region of the accretion disk, including the white dwarf, is just barely visible with a rim thickness of $h_{\mathrm{r}}=0.08$. Any "thick disk" models characterized by rim height $h_{\mathrm{r}}>0.08$ would result in complete shadowing of the white dwarf and part of the disk at all orbital phases, and thus would be insensitive to the white dwarf's luminosity.

Wood et al. also considered an alternative model with a disk rim, and found that their rim model did not result in a significantly steeper brightness temperature gradient. However, the rim model they considered was considerably less sophisticated than ours. In particular, their models employed a "dark" disk rim solely to study the effect produced by shadowing of the disk by the rim. The effect of the rim's contribution to the overall disk light was not included in their models. Furthermore, rim heights of only $1-2 \%$ of the disk radius were considered, which required inclination angles, $i>89^{\circ}$, to achieve significant rim shadowing.

As a test of the importance of a bright rim versus a dark rim like that considered by Wood et al., we ran a series of models with our standard flared disk, but with the rim brightness switched off. These "dark rim" models generally produced the same best-fit solutions as our standard models, but with one difference: a significantly lower value of the disk radial temperature parameter, $\alpha$. Specifically, for our four standard models with 
$\mathrm{a} \operatorname{rim}\left(q=0.2, h_{\mathrm{r}}=0.04 ; q=0.2, h_{\mathrm{r}}=0.08 ; q=0.4, h_{\mathrm{r}}=0.04 ; q=0.4, h_{\mathrm{r}}=0.08\right)$, we found values of $\alpha=0.15,0.45,0.25$, and 0.35 , respectively, compared with the values of $\alpha=0.35,0.75,0.25$, and 0.45 from our bright rim models (see Table 7 ). The only case where the bright rim apparently made no difference was in the model characterized by $q=0.4, h_{\mathrm{r}}=0.04$. However, in this case, the lower inclination and modest rim height make the contribution of the rim to the total disk light the smallest of all models considered.

The importance of a bright disk rim can be best visualized by referring to Figures 7 and 8, which show the system geometry at mid-eclipse. At high inclinations the disk rim makes a significant contribution to the total disk light. Since the disk rim (excluding the bright spot) has a nearly constant surface brightness, the eclipse of this rimmed disk will be more $\mathbf{V}$-shaped than the eclipse of a flat disk, even in the case where the disk has a steadystate $(\alpha=0.75)$ radial temperature profile (e.g., see the eclipse simulations and discussion of DW UMa by Knigge et al. 2000, 2004). As mentioned earlier, Smak (1994) has shown that modeling such eclipses under the assumption that the disk is flat (as in eclipse mapping) leads to the spurious conclusion that the disk temperature profile is shallower that $\alpha=0.75$. In the case of V Per, an additional effect is that for both mass ratios considered, the accretion disk is never completely eclipsed. In models with a disk rim, the contribution of the rim to the total system light at mid-eclipse can be significant. In order to produce an eclipse with the observed depth (in the normalized light curves), the inclusion of a bright outer disk rim requires that the eclipsed inner disk be sufficiently bright to compensate, i.e. to keep the ratio of the mid-eclipse to out-of-eclipse system brightness unchanged. For a given outer disk temperature, this can only be accomplished by having a luminous white dwarf, or by having a steep disk temperature profile, or both. These two possibilities produce very different ingress and egress eclipse profiles, and can therefore be distinguished by the model fit. In the case of $\mathrm{V}$ Per, the steep ingress and egress characteristic of a significant white dwarf contribution to the overall luminosity is not observed, leaving a steep disk temperature gradient as the only viable alternative.

\subsection{Correlations Between Model Parameters}

Further insights into the the effect that the various input parameters have on acceptable model solutions can be found by exploring correlations between the parameters. We begin by considering an expanded set of model solutions defined by $\chi_{\nu}^{2}<2.0$, which allows potential correlations to be studied over an expanded range in parameter space. Correlations between pairs of parameters are explored by allowing these parameters to vary while holding the remaining parameters fixed at their optimum values. For a total of five fitting parameters, 
there are a total of 10 such pairings. As a representative example, the correlations for the best-fitting $q=0.2, h_{\mathrm{r}}=0.04$ model are shown in Figure 9.

As in previous studies of GY Cnc and EX Dra (Papers I and II), the most highly correlated pairs involve $R_{\mathrm{d}}, T_{\mathrm{d}}$, and $\alpha$, which together determine the disk luminosity. There is a major difference in the correlations found in the present study, however. In the cases of GY Cnc and EX Dra, which are longer orbital period systems with larger mass ratios, the accretion disks were entirely occulted at mid-eclipse. Thus, the depth of eclipse fixes the disk luminosity. For a constant disk luminosity, the radial temperature parameter $\alpha$ should be negatively correlated with the temperature at the disk periphery, $T_{\mathrm{d}}$, and the disk radius, $R_{\mathrm{d}}$, as was indeed observed for these two systems. In the case of V Per, however, the situation is more complicated as described in the previous section. Because the disk is never completely obscured, the eclipse depth no longer uniquely defines the disk luminosity. The normalized eclipse depth can be achieved with a relatively low-luminosity disk having a relatively shallow radial temperature gradient, or with a disk having a bright rim (relatively high temperature and/or vertical extent) and steep temperature gradient (large $\alpha$ ). As the contribution of the disk rim is increased, the inner disk luminosity must increase to compensate, requiring a steeper temperature gradient and a higher value of $\alpha$. Thus, for a given disk radius and rim thickness, $\alpha$ should be positively correlated with the outer disk (rim) temperature, $T_{\mathrm{d}}$, as observed in the case of V Per, and shown in Figure 9. In view of these considerations, it is not surprising that the $q=0.40, h_{\mathrm{r}}=0.00$ and $q=0.20, h_{\mathrm{r}}=0.08$ models yield the least luminous and most luminous disks, respectively.

In principle, a smaller disk with the appropriate luminosity that is completely eclipsed could produce the observed eclipse depth, however, the shape of the eclipse profile, not just its depth is important for a good model fit. In the case of $\mathrm{V}$ Per, the maximum width of the eclipse profile (as defined by the onset of ingress and the termination of egress) requires a relatively large disk radius that approaches the tidal limit (see Figure 6).

\subsection{Model Constraints from the Disk Luminosity}

Although a range of models can produce acceptable fits to the data, as noted above, these models differ in one important respect: they represent significantly different disk luminosities. Referring to our best-fitting model solutions (Table 7), we are reminded that rimless models are characterized by flat temperature profiles (small $\alpha$ ). For a given outer disk radius and temperature, disks with relatively flat temperature gradients will be considerably less luminous than steady-state (rimmed) disks characterized by $\alpha=0.75$. Thus, a potential way to constrain the models further is to favor those models that produce the 
expected disk luminosity, and absolute magnitude for V Per. The disk luminosities can be explored through two independent approaches: directly from our model fluxes, and from an estimate of the mass accretion rate inferred from our models.

In the first approach, we can compare the ratio of our model $V$ fluxes for the disk and secondary star as viewed from an inclination angle of $i=0$ (i.e. "face-on"). This quantity, $\eta_{\mathrm{d}, \mathrm{s}}=F_{\mathrm{V}}^{\mathrm{d}}(i=0) / F_{\mathrm{V}}^{\mathrm{s}}(i=0)$, is given in Table 10 for our best-fitting models. If we ignore limb-darkening and assume a spherical secondary star and a flat circular disk, then the luminosity ratio is approximately equal to half the model flux ratio (a flat disk has half the surface area of a sphere). Thus, $L_{\mathrm{V}}^{\mathrm{d}} / L_{\mathrm{V}}^{\mathrm{s}} \simeq \eta_{\mathrm{d}, \mathrm{s}} / 2$, and an estimate of the secondary star's luminosity will yield a crude estimate for the disk luminosity. In reality, this estimate will be a slight underestimate of the disk luminosity because we have neglected the relatively small contribution of the rim to the overall disk luminosity. As an example, the flux ratio of our best-fitting $q=0.2, h_{\mathrm{r}}=0.08$ model is $\eta_{\mathrm{d}, \mathrm{s}}=3.18 \times 10^{3}$, which represents a luminosity ratio of $\sim 1.59 \times 10^{3}$. Thus, we estimate the disk in this model to be $\sim 8.0 \mathrm{mag}$ brighter than the secondary star. An estimate of $M_{\mathrm{V}}(2)$ can be found given the secondary star's spectral type, which we estimated earlier to be $\sim \mathrm{M} 4 \mathrm{~V}-\mathrm{M} 5 \mathrm{~V}$. For a main-sequence secondary star of radius $R_{2}=0.24 \mathrm{R}_{\odot}$ (Table 6) and spectral type M4V-M5V, we find $12.4<M_{\mathrm{V}}(2)<12.8$ (Popper 1980). Thus, for this model, we estimate an absolute visual magnitude for the accretion disk, and in effect for the V Per system (the white dwarf and secondary star contribute little $V$ light compared to the disk), to be $M_{\mathrm{V}} \simeq 4.6$. Although somewhat bright for a $P=2.6 \mathrm{hr}$ system, this value is certainly plausible for $\mathrm{V}$ Per, and is typical for the absolute visual magnitude of nova remnants (e.g. Warner 1995).

As a check on this result, we can also make a rough estimate the disk luminosity from an estimate of the mass transfer rate coupled with the $\dot{M}$ vs. $M_{\mathrm{V}}$ relation from Tylenda (1981). We begin with a well-known expression for the effective temperature of the accretion disk (e.g. Pringle 1981):

$$
\sigma T^{4}(r)=\frac{3 G M_{1} \dot{M}}{8 \pi r^{3}}\left[1-\sqrt{\frac{R_{\mathrm{in}}}{r}}\right],
$$

where $R_{\text {in }}$ is the inner radius of the disk. By adopting the model radius and temperature of the outer disk (rim), we can compute the mass accretion rate for a given model. Specifically,

$$
\dot{M}=\frac{8 \pi R_{\mathrm{d}}^{3} \sigma T_{\mathrm{d}}^{4}}{3 G M_{1}}\left[1-\sqrt{\frac{R_{\mathrm{in}}}{r}}\right]^{-1} \mathrm{gm} \mathrm{s}^{-1} .
$$

Mass transfer rates $\left(\dot{M}_{17}=\dot{M} / 10^{17} \mathrm{~g} \mathrm{~s}^{-1}\right)$ for our best-fitting models are given in Table 10 . For the specific case of the $q=0.2, h_{\mathrm{r}}=0.08$ model with $\alpha=0.75$, the mass-transfer rate can be converted to an equivalent $M_{\mathrm{V}}$ through an extrapolation of the relation given in 
Table 1 of Tylenda (1981). ${ }^{2}$ We find a value of $M_{\mathrm{V}} \simeq 4.3$, which is consistent with the value of $M_{\mathrm{V}} \simeq 4.6$ estimated earlier from the disk-to-secondary star luminosity ratio.

The absolute magnitudes and accretion rates of systems with flat accretion disks $\left(M_{\mathrm{V}} \simeq\right.$ $7-8$ and $\left.\dot{M}_{17} \simeq 1.5-2.5\right)$ are more typical of dwarf nova disks at minimum light. Such feeble disks with their shallow temperature gradients $(\alpha=0.15)$ are unlikely to be seen in nova and nova-like systems with stable accretion unless a significant amount of mass and accretion energy is being lost from the inner disk, for example, by driving a wind. In such a case, the mass accretion rate in the outer disk could possibly be increased without necessarily producing a luminous disk with a steep temperature profile.

Since V Per does not exhibit dwarf nova eruptions, a further constrain on possible models for $\mathrm{V}$ Per is provided by the requirement that the mass transfer rate exceed the citical value for stable accretion, $\dot{M}_{\text {crit }}$. A convenient expression for the critical mass transfer rate is given by $\dot{M}_{\text {crit }} \simeq 10^{16} R_{10}^{21 / 8} M_{1}^{-7 / 8} \mathrm{gm} \mathrm{s}^{-1}$, where $R_{10}$ is the radius of the disk in units of $10^{10} \mathrm{~cm}$ (e.g. see Shafter et al. 1986, and references therein). Values of $\dot{M}_{\text {crit }}$ for our best-fitting models are given in Table 10. The flat disk models have mass accretion rates that are significantly below the critical value, while only the thick disk models have rates clearly in excess of the critical value.

To minimize the number of fitting parameters in our models, we have set the disk rim temperature equal to the minimum temperature in the outer disk, $T_{\mathrm{d}}$. It seems possible, however, that this assumption overestimates the value of $T_{\mathrm{d}}$ used to compute the disk temperature profile since energy input from the mass-transfer stream is likely to raise the temperature of the disk rim over that appropriate for the outer disk region. If so, then our estimates of mass transfer rates and disk luminosities are best considered upper limits. In this case, the flat disk models with shallow temperature gradients become even more problematic, and our conclusion favoring the high-mass-accretion-rate, high-luminosity, thick-disk model for the V Per system is strengthened.

\subsection{The Distance to V Per}

The distance to V Per can be estimated by comparing the observed brightness at mideclipse with estimates of the absolute magnitude of the secondary star, and the fraction of

\footnotetext{
${ }^{2}$ It has been necessary to apply a small correction $(\sim 0.8 \mathrm{mag})$ to the resulting absolute magnitude to account for the difference in $M_{1}$ and $R_{\mathrm{d}}$ between Tylenda's model $\left(M_{1}=1 M_{\odot}, R_{\mathrm{d}}=5 \times 10^{10} \mathrm{~cm}\right)$ and our model. Following Warner (1995), the correction for $R_{\mathrm{d}}$ has been estimated from the models of Wade (1984).
} 
light it contributes at mid-eclipse. The fraction of light contributed by the secondary star at mid-eclipse, $f_{2}=f_{\mathrm{V}}^{\mathrm{s}}(\phi=0) / f_{\mathrm{V}}^{\text {tot }}(\phi=0)$, depends on the model considered, and in particular on the properties of the disk rim. Values of $f_{2}$ for each model are given in Table 10. In the rimless models, the disk contributes relatively little to the light at mid-eclipse compared with the models with rims. According to our best-fitting solutions for models with a thick rim, the secondary star contributes $\sim 1.0 \%$ of the light at mid-eclipse, while in the rimless models, where the disk luminosity is relatively low, the secondary star contributes a substantial $\sim 20 \%$ of the mid-eclipse light. Given that $m_{\mathrm{V}}=19.34$ (see Table 3 ) at mid-eclipse, we estimate the apparent distance moduli $(m-M)_{\mathrm{V}}$ given in Table 10.

The interstellar extinction along the line of sight can be estimated from the color excess obtained from a comparison of the observed and model $B-V$ colors. V Per has an observed color of $B-V \simeq 0.6 \pm 0.2$ outside of eclipse, where much of the uncertainty is due to the limited phase coverage of our outside eclipse $B$-band data. Assuming a ratio of total-toselective extinction of $R_{\mathrm{V}}=3$ yields the visual extinctions, $A_{\mathrm{V}}$, along the line of sight to $\mathrm{V}$ per given in Table 10. Our models with a rim have colors outside of eclipse that are significantly bluer than the colors of the rimless models, leading to larger values of $A_{\mathrm{V}}$. Correcting the apparent distance moduli yields the model-dependent distance estimates given in Table 10.

As a consistency check, the distance to V Per can also be determined from a comparison of the out-of-eclipse magnitude with an estimate of the system's absolute magnitude corrected for inclination. Warner $(1987,1995)$ gives a correction factor for the conversion of absolute magnitude to apparent absolute magnitude of $\Delta M_{\mathrm{V}}(i)=-2.5 \log [(1+1.5 \cos i) \cos i]$; however, this correction does not take into account any contribution from the disk rim. ${ }^{3}$ Fortunately, the ratio of rim-to-disk light $\left(\eta_{\mathrm{r}, \mathrm{d}}\right)$ is given by our models (see Table 10 ). In the case of our favored $q=0.2, h_{\mathrm{r}}=0.08$ model, the rim contributes $\sim 50 \%$ of the disk light. Applying these corrections for inclination, taking $M_{\mathrm{V}}=4.6$ for the $q=0.2, h_{\mathrm{r}}=0.08$ model, and adopting $m_{\mathrm{V}}=18.1$ for V Per out of eclipse (see Table 3), yields a reddening-corrected distance modulus of $(m-M)_{\mathrm{V}}=9.6$, and a corresponding distance of $\sim 820 \mathrm{pc}$. Given the uncertainties, this value is consistent with our estimate of $1 \mathrm{kpc}$ based on the properties of the secondary star.

In summary, we estimate that $\mathrm{V}$ Per lies at a distance of $\sim 1.0 \mathrm{kpc}$, in our favored scenario where the disk has a significant rim that contributes to the light at mid-eclipse. In contrast, our rimless models, which predict that a significantly larger fraction of the light at mid-eclipse arises from the secondary star, suggest that V Per lies at a distance of

\footnotetext{
${ }^{3}$ The expression given in Warner (1995) corrects an error in the Warner (1987) formula, however the sign is incorrect for his stated conversion of apparent absolute magnitude to absolute magnitude.
} 
only $\sim 0.5 \mathrm{kpc}$. In both cases, a significant component of the uncertainty in our distance estimates arises from uncertainties in the visual extinction. Overall, our favored distance estimate is consistent with the value of $\sim 1 \mathrm{kpc}$ derived by Shafter (1997) from a comparison of the apparent magnitude at maximum light $\left(m_{\max } \simeq 4\right)$, an assumed absolute magnitude at maximum light, $M_{\mathrm{V}}=-7.5$, and a visual extinction of $A_{\mathrm{V}}=1.4$ estimated from the reddening maps of Neckel et al. (1980).

\section{Conclusions}

We have performed the first multicolor eclipse study of the classical nova V Per using a parameter-fitting eclipse code to model the light curve. Our study has explored a variety of assumptions regarding the structure of the accretion disk, including models with a large inner disk radius, as might be expected if $\mathrm{V}$ Per has a strongly magnetic white dwarf, and models that include a disk rim. In agreement with the earlier monochromatic maximum entropy eclipse mapping study of V Per by Wood et al. (1992), we find that a flat accretion disk that extends down to the surface of the white dwarf must have a brightness temperature profile that is significantly flatter than that expected for a steady-state disk. In an attempt to resolve the discrepancy, Wood et al. explored models where the inner disk was disrupted, as might be expected if V Per harbors a magnetic white dwarf. They found that temperature profiles consistent with the steady-state case could be produced in models with an increased inner disk radius, $R_{\mathrm{in}} \simeq 0.2 R_{\mathrm{L} 1}$.

Although the brightness temperature profiles of our best-fitting models become steeper when the inner disk is truncated out to $R_{\text {in }}=0.2 R_{\mathrm{L} 1}$, unlike Wood et al., we were unable to reproduce a flat-disk model with $\alpha \simeq 0.75$ with a truncated inner disk alone. However, our models that included a disk rim were characterized by considerably steeper brightness temperature profiles, particularly in our "thick disk" $\left(h_{\mathrm{r}}=0.08\right)$ case where we found $\alpha=0.75$ gives the best fit to the data. Although acceptable fits for models with rims can be found with smaller values of $\alpha$ and lower outer disk temperatures (faint rims), the only way to achieve $\alpha=0.75$ is with a model having a bright rim. Furthermore, only the bright rim models give accretion rates and absolute magnitudes that are typical of nova systems with stable accretion. By extrapolating our result for V Per, we confirm the conclusion reached by Smak (1994) from his generic eclipse simulations, that prior attempts to analyze cataclysmic variable eclipses with flat-accretion-disk models have significantly underestimated the brightness temperature gradients of the accretion disks.

In summary, regardless of the adopted mass ratio or rim thickness, we find that V Per is most likely characterized by a large accretion disk (extending to near its tidal radius), 
characterized by $R_{\mathrm{d}} \simeq 0.75 R_{\mathrm{L} 1}$, with a temperature $T_{\mathrm{d}} \simeq 6000-10000 \mathrm{~K}$ at its outer edge. No evidence was found for an unusually luminous (hot) white dwarf or bright spot in the system. However, if the disk is in a steady-state, optically-thick configuration, we have shown that the disk must possess a significant rim. As in V Per, it is also likely that other short-period cataclysmic variable stars with mass ratios, $q \lesssim 0.5$, will also potentially harbor accretion disks that are not fully occulted at mid-eclipse. As shown in the present study, it is imperative that any attempts to model the accretion disks in these systems should take into account the effect of a disk rim.

We thank the staff of the Steward Observatory for granting time on the Bok $2.3 \mathrm{~m}$ reflector and Karl Gordon for assistance with the observations. We also thank John Cannizzo and Ted Daub for reading an earlier version of the manuscript and for subsequent discussion, Jerry Orosz for computing pictorial representations of the system geometry near mid-eclipse, and the referee, I. B. Bíró, for a thorough report that improved our presentation. We are also grateful to Christian Knigge and Jean-Pierre Lasota for bringing an important reference omitted in the original manuscript to our attention. Our eclipse model is based in part on occultation kernel subroutines kindly provided by K. Horne.

\section{REFERENCES}

Bíró, I. B. 2000, A\&Ap, 364, 573.

Cannizzo, J. K. 2001, ApJ, 556, 847.

Cannizzo, J. K. \& Wheeler, J. C. 1984, ApJS, 55, 367.

Chanan, G. A., Middleditch, J. \& Nelson, J. E. 1976, ApJ, 208, 512.

Dahm, S. 1997, Master's Thesis, San Diego State University.

Horne, K. 1985, MNRAS, 213, 129.

Katysheva, N. A., Pavlenko, E. P., \& Shugarov, S. Y. 2002 in Classical Nova Explosions. AIP Conference Proceedings, Vol 637, eds M. Hernanz and J. José, 543.

Knigge, C., Long, K. S., Howard, D. W., Szkody, P., \& Dhillon, V. S. 2000, ApJ, 539, L49.

Knigge, C., Araujo-Betancor, S., Gänsicke, B. T., Long, K. S., Szkody, P., Howard, D. W., Hynes, R. I. \& Dhillon, V. S. 2004, ApJ, 615, L129.

Kolb, U. 1996, in Cataclysmic Variables \& Related Objects, eds. A. Evans and J. H. Wood (Kluwer, Dordrecht), 433.

Matthews, T. A. \& Sandage, A. R. 1963, Ap. J., 138, 30. 
McLaughlin, D. B., 1946, PASP, 58, 218.

Meyer, F., \& Meyer-Hofmeister, E. 1982, A\&A, 106, 34.

Neckel, Th., Klare, G., \& Sarcander, M. 1980, A\&AS, 42, 251.

Pringle, J. E. 1981, Ann. Rev. Astr. Ap., 19, 137.

Politano, M. 1988, Ph.D. Thesis, University of Illinois.

Popper D. M. 1980, Ann. Rev. Astr. Ap., 18, 115.

Ritter, H. 1996, in Evolutionary Processes in Binary Stars, eds. R. A. M. J Wijers, M. B. Davies, and C. A. Tout (Kluwer, Dordrecht), p.223.

Robinson, E. L., Wood, J. H., \& Wade, R. A. 1999, ApJ, 514, 952.

Rutten, R. G. M., van Paradijs, J., Tinbergen, J. 1992, A\&Ap, 260, 213.

Shafter, A. W. 1997, ApJ, 487, 226.

Shafter, A. W. \& Abbott, T. M. C. 1989, ApJ, 339, L75.

Shafter, A. W., Clark, L. L., Holland, J. N., \& Williams, S. J. 2000, PASP, 112, 1467 (Paper I).

Shafter, A. W. \& Holland, J. N. 2003, PASP, 115, 1105 (Paper II).

Shafter, A. W., Wheeler, J. C., \& Cannizzo, J. K. 1986, ApJ 305, 261.

Shakura, N. I. \& Sunyaev, R. A. 1973, A\&A, 24, 337.

Smak, J. 1992, AcA, 42, 323.

Smak, J. 1994, AcA, 44, 265.

Smith, D. A., \& Dhillon, V. S. 1998, MNRAS, 301, 767.

Sulkanen, M. E., Brasure, L. W., \& Patterson, J. 1981, ApJ, 244, 579.

Tylenda, R. 1981, AcA, 31, 127.

Wade, R. A. 1984, MNRAS, 208, 381.

Warner, B. 1995, Cataclysmic Variable Stars, Cambridge Univ. Press.

Warner, B. 1987, MNRAS, 227, 23.

Wood, J. H., Abbott, T. M. C. \& Shafter, A. W. 1992, ApJ, 393, 729.

van Hamme, W. 1993, AJ, 106, 2096 
Table 1. CVs in the Period Gap

\begin{tabular}{lcc}
\hline \hline \multicolumn{1}{c}{ Name } & Type & Orbital Period (hr) \\
\hline V516 Pup & AM & 2.29 \\
SDSS J205017-053627 & NL & 2.30 \\
QS Tel & AM & 2.33 \\
DD Cir & N, DQ & 2.34 \\
NY Ser & SU & 2.35 \\
SDSS J080908+381406 & NL & 2.38 \\
V348 Pup & DQ & 2.44 \\
MN Dra & SU & 2.50 \\
RX J1554.2+2721 & AM & 2.53 \\
V Per & N & 2.57 \\
V795 Her & DQ & 2.60 \\
1RXS J052430+424449 & AM, DQ & 2.62 \\
V349 Pav & AM & 2.66 \\
QU Vul & N & 2.68 \\
SDSS J075240+362823 & AM? & 2.70 \\
\hline
\end{tabular}


Table 2. Summary of Observations

\begin{tabular}{ccccc}
\hline \hline UT Date & $\begin{array}{c}\text { UT Time } \\
\text { (start of observations) }\end{array}$ & $\begin{array}{c}\text { Time Resolution } \\
\text { (sec) }\end{array}$ & $\begin{array}{c}\text { Number of } \\
\text { Exposures }\end{array}$ & Filter \\
\hline 2002 Oct 10 & $4: 57: 00$ & 41.5 & 371 & V \\
2002 Oct 10 & $10: 34: 00$ & 41.5 & 151 & V \\
2002 Oct 11 & $7: 05: 00$ & 57.0 & 248 & B \\
2002 Oct 29 & $4: 33: 00$ & 56.1 & 74 & $\mathrm{R}$ \\
2002 Oct 29 & $7: 00: 00$ & 56.1 & 74 & $\mathrm{R}$ \\
2002 Oct 29 & $9: 11: 38$ & 56.2 & 121 & $\mathrm{I}$ \\
2002 Dec 09 & $2: 20: 35$ & 56.4 & 111 & $\mathrm{I}$ \\
2002 Dec 09 & $5: 22: 00$ & 56.4 & 77 & $\mathrm{~B}$ \\
2002 Dec 09 & $7: 32: 28$ & 56.4 & 96 & $\mathrm{I}$ \\
2002 Dec 11 & $5: 17: 00$ & 56.4 & 132 & $\mathrm{R}$ \\
\hline
\end{tabular}

${ }^{a}$ Mean time interval between exposures (Integration time plus readout time) 
Table 3. Mean Magnitudes and Colors

\begin{tabular}{|c|c|c|c|}
\hline $\begin{array}{c}\text { Photometric } \\
\text { Parameter }\end{array}$ & $\begin{array}{c}\text { Effective } \\
\text { Wavelength } \\
(\AA)\end{array}$ & Out of Eclipse ${ }^{\mathrm{a}}$ & Primary Minimum \\
\hline$\ldots \ldots$ & 4375 & $18.7 \pm 0.1$ & $20.05 \pm 0.09$ \\
\hline$V \ldots \ldots \ldots$ & 5460 & $18.1 \pm 0.1$ & $19.34 \pm 0.08$ \\
\hline$R \ldots \ldots \ldots$ & 6543 & $18.1 \pm 0.2$ & $19.13 \pm 0.31$ \\
\hline$I \ldots \ldots \ldots$ & 7992 & $17.5 \pm 0.1$ & $18.61 \pm 0.17$ \\
\hline$B-V \ldots$ & $\ldots$ & $0.6 \pm 0.2$ & $0.71 \pm 0.12$ \\
\hline$V-R \ldots$ & $\ldots$ & $0.0 \pm 0.3$ & $0.21 \pm 0.32$ \\
\hline$V-I \ldots$ & $\ldots$ & $0.6 \pm 0.2$ & $0.73 \pm 0.19$ \\
\hline
\end{tabular}

${ }^{a}$ The mean magnitude of the light curve excluding orbital phases in the range: $-0.15<\phi<0.15$. 
Table 4. Eclipse Timings

\begin{tabular}{ccccc}
\hline \hline $\begin{array}{c}\text { HJD (mid-eclipse) } \\
(2,400,000+)\end{array}$ & $\begin{array}{c}\text { Cycle Number } \\
(E)\end{array}$ & Filter & $\begin{array}{c}O-C \\
\left(\times 10^{3} \text { day }\right)\end{array}$ & reference $^{\mathrm{a}}$ \\
\hline $47442.8264 \ldots$ & 0. & BVR & 0.438541 & 1 \\
$47442.9337 \ldots$ & 1. & BVR & 0.615067 & 1 \\
$47443.7896 \ldots$ & 9. & BVR & -0.472726 & 1 \\
$47443.8965 \ldots$ & 10. & BVR & -0.696200 & 1 \\
$47444.0040 \ldots$ & 11. & BVR & -0.319674 & 1 \\
$47446.8969 \ldots$ & 38. & BVR & 0.246525 & 1 \\
$47447.0038 \ldots$ & 39. & BVR & 0.023051 & 1 \\
$47448.9321 \ldots$ & 57. & BVR & 0.100518 & 1 \\
$50430.7140 \ldots$ & 27892. & V & 0.099267 & 2 \\
$50430.8207 \ldots$ & 27893. & V & -0.324207 & 2 \\
$50481.5978 \ldots$ & 28367. & R & 0.249075 & 2 \\
$50481.7052 \ldots$ & 28368. & B & 0.525601 & 2 \\
$50509.6639 \ldots$ & 28629. & V & -0.001136 & 2 \\
$50692.8455 \ldots$ & 30339. & B & 0.458173 & 2 \\
$50695.8445 \ldots$ & 30367. & R & 0.000898 & 2 \\
$50696.9157 \ldots$ & 30377. & B & -0.033843 & 2 \\
$50696.8089 \ldots$ & 30376. & R & 0.289631 & 2 \\
$50730.7668 \ldots$ & 30693. & I & 0.048345 & 2 \\
$50730.8740 \ldots$ & 30694. & I & 0.124871 & 2 \\
$50730.9807 \ldots$ & 30695. & $\mathrm{I}$ & -0.298603 & 2 \\
$50731.9451 \ldots$ & 30704. & $\mathrm{~V}$ & -0.009870 & 2 \\
\hline
\end{tabular}


Table 4 (cont.). Eclipse Timings

\begin{tabular}{cccrc}
\hline \hline $\begin{array}{c}\text { HJD (mid-eclipse) } \\
(2,400,000+)\end{array}$ & $\begin{array}{c}\text { Cycle Number } \\
(E)\end{array}$ & Filter & $\begin{array}{c}O-C \\
\left(\times 10^{3} \text { day }\right)\end{array}$ & ref a $^{\text {a }}$ \\
\hline $50757.8691 \ldots$ & 30946. & $\mathrm{R}$ & 0.109401 & 2 \\
$50758.7253 \ldots$ & 30954. & $\mathrm{I}$ & -0.678392 & 2 \\
$50758.8331 \ldots$ & 30955. & $\mathrm{~B}$ & -0.001866 & 2 \\
$50759.7971 \ldots$ & 30964. & $\mathrm{~B}$ & -0.113133 & 2 \\
$50759.9041 \ldots$ & 30965. & $\mathrm{~V}$ & -0.236607 & 2 \\
$52172.541 \ldots$ & 44152. & $\mathrm{R}$ & -0.589411 & 3 \\
$52557.7580 \ldots$ & 47748. & $\mathrm{~V}$ & 0.397768 & 4 \\
$52557.8642 \ldots$ & 47749. & $\mathrm{~V}$ & -0.525707 & 4 \\
$52557.9720 \ldots$ & 47750. & $\mathrm{~V}$ & 0.150819 & 4 \\
$52558.8293 \ldots$ & 47758. & $\mathrm{~B}$ & 0.463027 & 4 \\
$52558.9362 \ldots$ & 47759. & $\mathrm{~B}$ & 0.239553 & 4 \\
$52576.7186 \ldots$ & 47925. & $\mathrm{R}$ & 0.142854 & 4 \\
$52576.8255 \ldots$ & 47926. & $\mathrm{R}$ & -0.080620 & 4 \\
$52576.9323 \ldots$ & 47927. & $\mathrm{I}$ & -0.404094 & 4 \\
$52617.6398 \ldots$ & 48307. & $\mathrm{I}$ & 0.175752 & 4 \\
$52617.7469 \ldots$ & 48308. & $\mathrm{~B}$ & 0.152278 & 4 \\
$52617.8537 \ldots$ & 48309. & $\mathrm{I}$ & -0.171196 & 4 \\
$52619.7820 \ldots$ & 48327. & $\mathrm{R}$ & -0.093730 & 4 \\
\hline
\end{tabular}

a 1.- Shafter \& Abbott 1989; 2.- Dahm (1997); 3.- Katysheva et al. 2002; 4.- This work. 
Table 5. Model Input Parameters

\begin{tabular}{lcc}
\hline \hline Parameter & Definition & Value $^{\mathrm{a}}$ \\
\hline$P \ldots \ldots$ & Orbital Period, $P$ & $2.57 \mathrm{hr}$ \\
$q \ldots \ldots$ & Mass ratio, $M_{2} / M_{1}$ & $0.20,0.40$ \\
$\Delta \phi \ldots \ldots$ & Eclipse phase width & 0.073 \\
$i \ldots \ldots$ & Orbital inclination & computed \\
$\alpha \ldots \ldots$ & Disk temperature parameter & variable \\
$R_{\mathrm{d}} \ldots \ldots$ & Disk radius & variable \\
$R_{\mathrm{in}} \ldots \ldots$ & Inner disk radius & $R_{1}, 0.2 R_{\mathrm{L} 1}$ \\
$h_{\mathrm{r}} \ldots \ldots$ & Disk rim parameter & $0.00,0.04,0.08$ \\
$T_{\mathrm{d}} \ldots \ldots$ & Temperature of disk perimeter & variable \\
$R_{1} \ldots \ldots$ & Radius of white dwarf & computed \\
$R_{2} \ldots \ldots$ & Radius of secondary star & computed \\
$T_{1} \ldots \ldots$ & Temperature of white dwarf & variable \\
$T_{2} \ldots \ldots$ & Temperature of secondary star & $3300 \mathrm{~K}$ \\
$\chi_{\mathrm{s}} \ldots \ldots$ & Bright spot temperature factor & variable \\
$R_{s} \ldots \ldots$ & Bright spot radius & $0.2 R_{\mathrm{d}}$ \\
\hline
\end{tabular}

${ }^{a}$ Where given explicitly, the values are fixed and not varied during the fitting procedure. The values of "variable" parameters are determined by the model during the fitting procedure, while the "computed" values are calculated by the model for a given mass ratio. 
Table 6. Binary Parameters

\begin{tabular}{ccc}
\hline \hline Parameter & $q=0.20$ & $q=0.40$ \\
\hline$i\left(^{\circ}\right) \ldots \ldots \ldots$ & 85.4 & 79.4 \\
$a\left(R_{\odot}\right) \ldots \ldots \ldots$ & 0.95 & 0.80 \\
$R_{\mathrm{L} 1}(a) \ldots \ldots \ldots$ & 0.66 & 0.59 \\
$R_{1}\left(10^{-2} R_{\odot}\right) \ldots$ & 0.97 & 1.52 \\
$M_{1}\left(M_{\odot}\right) \ldots \ldots$ & 0.85 & 0.43 \\
$R_{2}\left(R_{\odot}\right) \ldots \ldots$ & 0.24 & 0.24 \\
$M_{2}\left(M_{\odot}\right) \ldots \ldots$ & 0.17 & 0.17 \\
\hline
\end{tabular}


Table 7. Model Grid Solutions ${ }^{\mathrm{a}}$

\begin{tabular}{lcccccc}
\hline \hline & \multicolumn{3}{c}{$q=0.20$} \\
Parameter & $h_{\mathrm{r}}=0.00$ & $h_{\mathrm{r}}=0.04$ & $h_{\mathrm{r}}=0.08$ & $h_{\mathrm{r}}=0.00$ & $\begin{array}{c}q=0.40 \\
h_{\mathrm{r}}=0.04\end{array}$ & $h_{\mathrm{r}}=0.08$ \\
\hline$R_{\mathrm{d}}(a)$ & $0.49(0.40)$ & $0.49(0.40)$ & $0.46(0.40)$ & $0.41(0.41)$ & $0.44(0.44)$ & $0.41(0.38)$ \\
$R_{\mathrm{d}}(a)_{\mathrm{lim}} \mathrm{b}$ & $0.50(0.50)$ & $0.50(0.50)$ & $0.50(0.50)$ & $0.43(0.43)$ & $0.43(0.43)$ & $0.43(0.43)$ \\
$R_{\mathrm{d}}\left(R_{\mathrm{L} 1}\right)$ & $0.65(0.60)$ & $0.75(0.65)$ & $0.75(0.70)$ & $0.70(0.65)$ & $0.75(0.70)$ & $0.75(0.75)$ \\
$\alpha$ & $0.15(0.35)$ & $0.35(0.55)$ & $0.75(0.75)$ & $0.15(0.25)$ & $0.25(0.45)$ & $0.45(0.65)$ \\
$T_{1}\left(10^{3} \mathrm{~K}\right)$ & $10.0(10.0)$ & $10.0(15.0)$ & $10.0(25.0)$ & $10.0(10.0)$ & $10.0(10.0)$ & $10.0(15.0)$ \\
$T_{\mathrm{d}}\left(10^{3} \mathrm{~K}\right)$ & $6.0(6.0)$ & $6.0(6.0)$ & $10.0(6.0)$ & $6.0(6.0)$ & $6.0(6.0)$ & $8.0(6.0)$ \\
$\chi_{\mathrm{s}}$ & $1.2(1.2)$ & $1.2(1.1)$ & $1.3(1.1)$ & $1.1(1.1)$ & $1.3(1.2)$ & $1.3(1.2)$ \\
$(B-V)_{\circ}$ & $0.63(0.56)$ & $0.45(0.44)$ & $0.03(0.34)$ & $0.59(0.56)$ & $0.49(0.45)$ & $0.19(0.33)$ \\
$\chi_{\nu, \min }^{2}$ & $1.7(1.7)$ & $1.6(1.6)$ & $1.5(1.6)$ & $1.6(1.6)$ & $1.5(1.6)$ & $1.5(1.5)$ \\
\hline
\end{tabular}

${ }^{a}$ Values in parenthesis represent intermediate polar models where the inner accretion disk has been truncated at $R_{\mathrm{d}}\left(R_{\mathrm{L} 1}\right)=0.2$.

${ }^{\mathrm{b}}$ The limiting tidal radius of the disk computed using eqn (2.61) of Warner (1995). 
Table 8. Standard Model Distribution Mean Solutions

\begin{tabular}{lcccccc}
\hline \hline & \multicolumn{3}{c}{$q=0.20$} \\
Parameter & $h_{\mathrm{r}}=0.00$ & $h_{\mathrm{r}}=0.04$ & $h_{\mathrm{r}}=0.08$ & $h_{\mathrm{r}}=0.00$ & $\begin{array}{c}q=0.40 \\
h_{\mathrm{r}}=0.04\end{array}$ & $h_{\mathrm{r}}=0.08$ \\
\hline$<\chi_{\nu}^{2}>$ & 1.74 & 1.61 & 1.59 & 1.74 & 1.66 & 1.58 \\
$R_{\mathrm{d}}\left(R_{\mathrm{L} 1}\right)$ & $0.67 \pm 0.05$ & $0.73 \pm 0.04$ & $0.73 \pm 0.03$ & $0.71 \pm 0.04$ & $0.73 \pm 0.03$ & $0.75 \pm 0.02$ \\
$\alpha$ & $0.17 \pm 0.09$ & $0.45 \pm 0.13$ & $0.60 \pm 0.13$ & $0.19 \pm 0.10$ & $0.32 \pm 0.11$ & $0.51 \pm 0.12$ \\
$T_{1}\left(10^{3} \mathrm{~K}\right)$ & $14.8 \pm 2.05$ & $23.0 \pm 4.44$ & $25.6 \pm 4.77$ & $14.7 \pm 4.52$ & $18.5 \pm 4.51$ & $24.0 \pm 5.84$ \\
$T_{\mathrm{d}}\left(10^{3} \mathrm{~K}\right)$ & $7.22 \pm 0.97$ & $8.76 \pm 1.91$ & $8.92 \pm 1.77$ & $8.15 \pm 1.68$ & $8.35 \pm 1.82$ & $10.2 \pm 2.40$ \\
$\chi_{\mathrm{s}}$ & $1.18 \pm 0.15$ & $1.27 \pm 0.11$ & $1.23 \pm 0.08$ & $1.14 \pm 0.14$ & $1.29 \pm 0.13$ & $1.32 \pm 0.11$ \\
$(B-V)_{\circ}$ & $0.35 \pm 0.15$ & $0.13 \pm 0.19$ & $0.08 \pm 0.14$ & $0.26 \pm 0.17$ & $0.18 \pm 0.19$ & $0.03 \pm 0.16$ \\
\hline
\end{tabular}


Table 9. Intermediate Polar Distribution Mean Solutions

\begin{tabular}{lcccccc}
\hline \hline & \multicolumn{3}{c}{$q=0.20$} \\
Parameter & $h_{\mathrm{r}}=0.00$ & $h_{\mathrm{r}}=0.04$ & $h_{\mathrm{r}}=0.08$ & $h_{\mathrm{r}}=0.00$ & $\begin{array}{c}q=0.40 \\
h_{\mathrm{r}}=0.04\end{array}$ & $h_{\mathrm{r}}=0.08$ \\
\hline$<\chi_{\nu}^{2}>$ & 1.77 & 1.67 & 1.69 & 1.73 & 1.65 & 1.62 \\
$R_{\mathrm{d}}\left(R_{\mathrm{L} 1}\right)$ & $0.65 \pm 0.05$ & $0.68 \pm 0.05$ & $0.65 \pm 0.05$ & $0.69 \pm 0.05$ & $0.72 \pm 0.04$ & $0.72 \pm 0.03$ \\
$\alpha$ & $0.42 \pm 0.15$ & $0.60 \pm 0.14$ & $0.60 \pm 0.15$ & $0.35 \pm 0.15$ & $0.55 \pm 0.16$ & $0.65 \pm 0.12$ \\
$T_{1}\left(10^{3} \mathrm{~K}\right)$ & $15.2 \pm 2.33$ & $23.8 \pm 4.11$ & $24.7 \pm 4.44$ & $13.2 \pm 2.57$ & $17.8 \pm 3.02$ & $24.1 \pm 4.42$ \\
$T_{\mathrm{d}}\left(10^{3} \mathrm{~K}\right)$ & $6.54 \pm 0.88$ & $6.66 \pm 0.94$ & $6.53 \pm 0.88$ & $6.82 \pm 0.98$ & $6.94 \pm 1.10$ & $7.36 \pm 1.31$ \\
$\chi_{\mathrm{s}}$ & $1.18 \pm 0.15$ & $1.16 \pm 0.08$ & $1.10 \pm 0.06$ & $1.15 \pm 0.14$ & $1.20 \pm 0.13$ & $1.18 \pm 0.10$ \\
$(B-V)_{\circ}$ & $0.30 \pm 0.10$ & $0.26 \pm 0.13$ & $0.30 \pm 0.12$ & $0.31 \pm 0.14$ & $0.23 \pm 0.16$ & $0.18 \pm 0.14$ \\
\hline
\end{tabular}


Table 10. Luminosities, Mass Accretion Rates, and Distances

\begin{tabular}{lcccccc}
\hline \hline & \multicolumn{3}{c}{$q=0.20$} \\
\multicolumn{1}{c}{ Parameter } & $h_{\mathrm{r}}=0.00$ & $h_{\mathrm{r}}=0.04$ & $h_{\mathrm{r}}=0.08$ & $h_{\mathrm{r}}=0.00$ & $\begin{array}{c}q=0.40 \\
h_{\mathrm{r}}=0.04\end{array}$ & $h_{\mathrm{r}}=0.08$ \\
\hline$\eta_{\mathrm{d}, \mathrm{s}}$ & $155(148)$ & $387(283)$ & $3178(493)$ & $98.4(83.4)$ & $148(146)$ & $622(277)$ \\
$\eta_{\mathrm{r}, \mathrm{d}}$ & $0.0(0.0)$ & $0.34(0.34)$ & $0.51(0.40)$ & $0.0(0.0)$ & $0.21(0.17)$ & $0.31(0.19)$ \\
$M_{\mathrm{V}}$ & $7.9(7.9)$ & $6.9(7.2)$ & $4.6(6.6)$ & $8.4(8.6)$ & $7.9(7.9)$ & $6.4(7.2)$ \\
$\dot{M}_{17}\left(\mathrm{~g} \mathrm{~s}^{-1}\right)$ & $1.49(2.34)$ & $2.26(2.83)$ & $17.4(3.38)$ & $1.68(2.37)$ & $2.05(2.84)$ & $6.47(3.36)$ \\
$\dot{M}_{\mathrm{crit}, 17}\left(\mathrm{~g} \mathrm{~s}^{-1}\right)$ & $1.72(2.34)$ & $2.50(1.72)$ & $2.50(2.09)$ & $1.82(1.50)$ & $2.20(1.82)$ & $2.20(2.20)$ \\
$f_{2}$ & $0.23(0.26)$ & $0.077(0.11)$ & $0.0089(0.056)$ & $0.16(0.19)$ & $0.088(0.096)$ & $0.022(0.051)$ \\
$(m-M)_{\mathrm{V}}$ & $8.34(8.20)$ & $9.52(9.14)$ & $11.87(9.87)$ & $8.73(8.54)$ & $9.38(9.29)$ & $10.88(9.97)$ \\
$A_{\mathrm{V}}$ & $0.0(0.12)$ & $0.45(0.30)$ & $1.71(0.78)$ & $0.03(0.12)$ & $0.33(0.45)$ & $1.23(0.81)$ \\
$d(\mathrm{kpc})$ & $0.47(0.42)$ & $0.65(0.59)$ & $1.08(0.66)$ & $0.55(0.48)$ & $0.65(0.59)$ & $0.85(0.68)$ \\
\hline
\end{tabular}




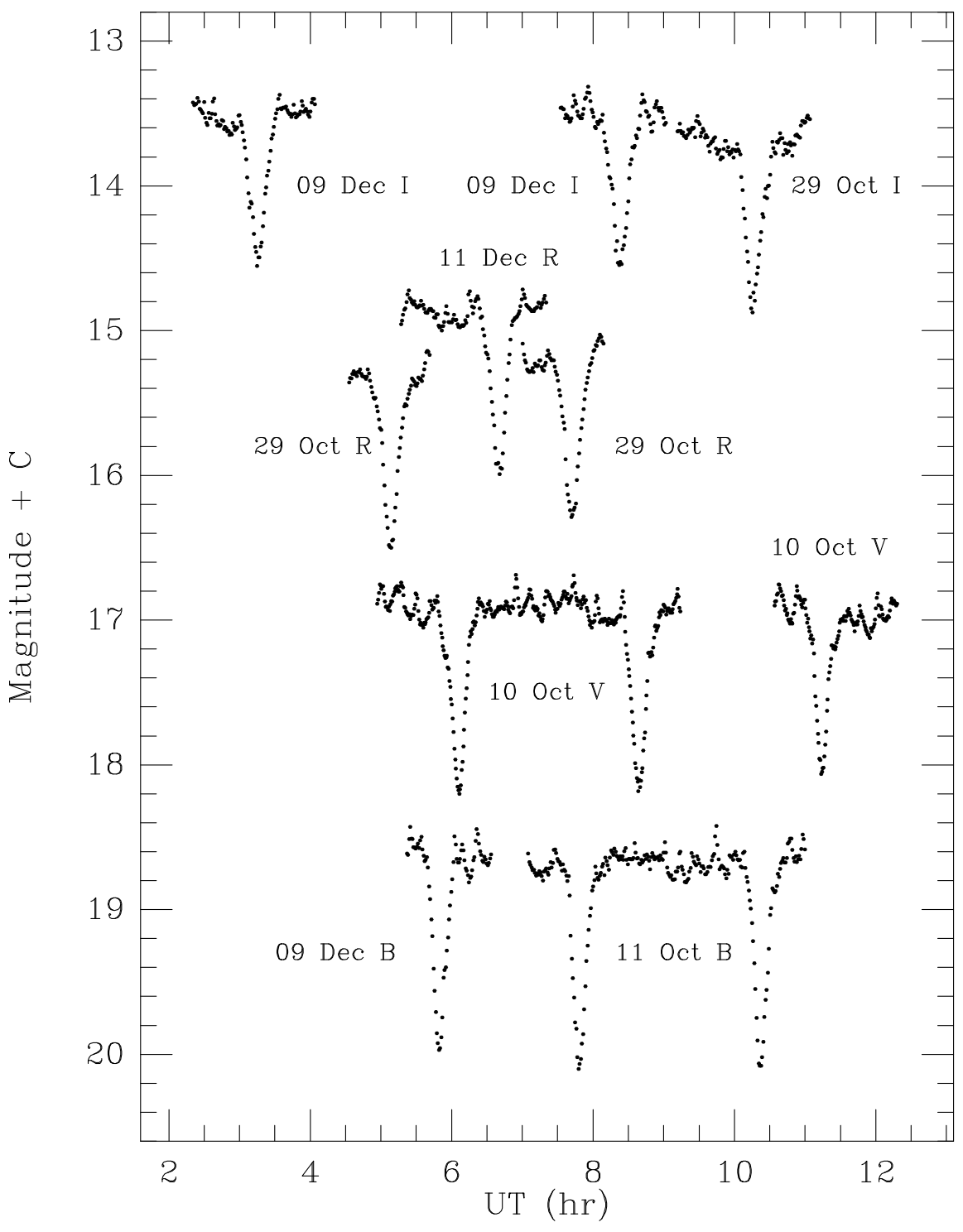

Fig. 1. - The multicolor light curves of V Per. For clarity of presentation, the data have been offset by addition of the following constants: $B$-band: $\mathrm{C}=0.0 ; V$-band: $\mathrm{C}=1.2 ; R$-band: $\mathrm{C}=2.4$; I-band: $\mathrm{C}=4.0$. 


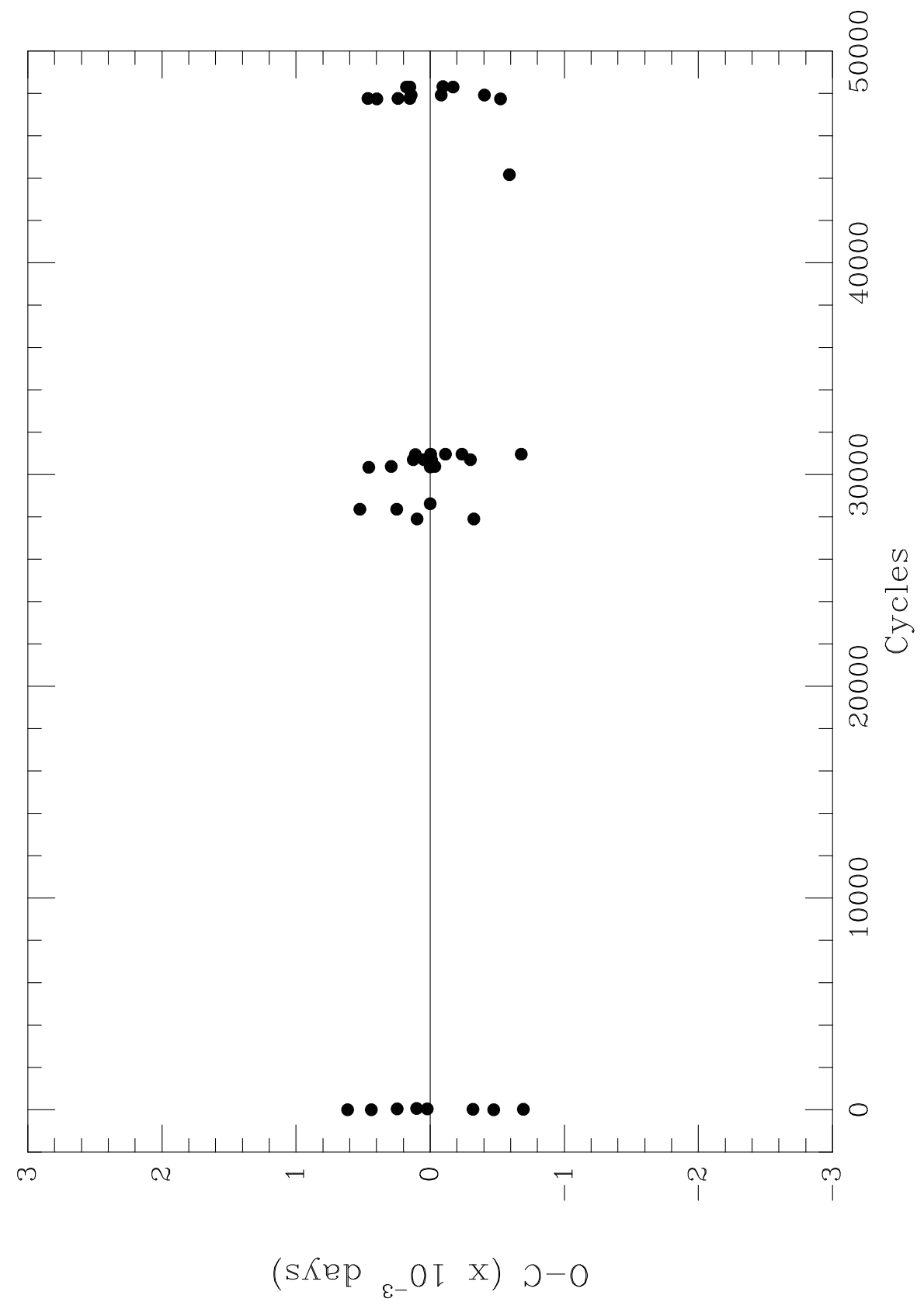

Fig. 2.- The residuals of the the observed times of mid-eclipse with respect to eqn (1) is plotted as a function of cycle number. The final grouping of points near cycle number 48000 are based on eclipse timings from this work, while the remaining points are based on timings from the literature. There is no evidence for any period change in V Per over the $\sim 15$ years of available eclipse timings. 

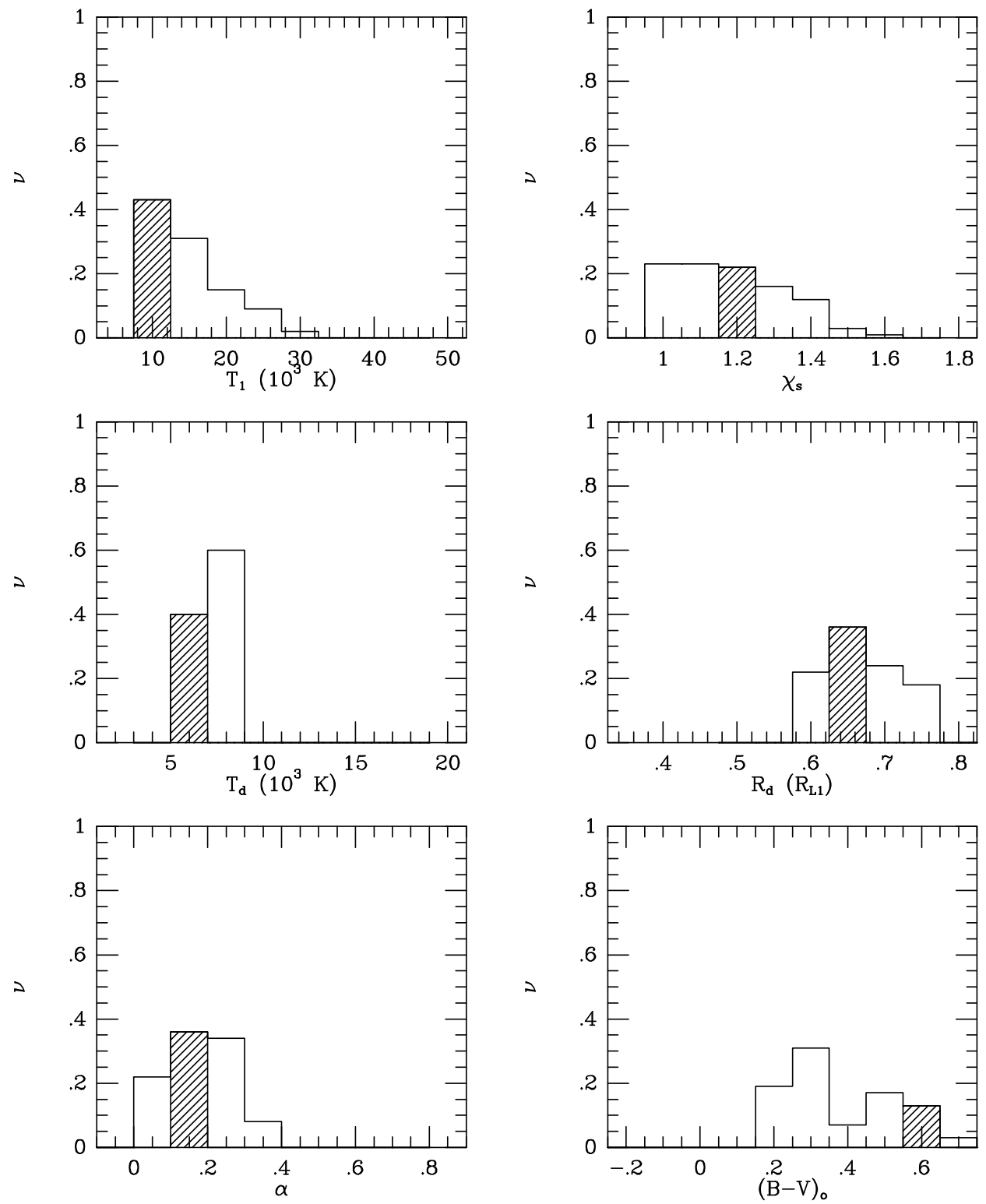

Fig. 3.- The $q=0.20, h_{\mathrm{r}}=0.00$ frequency distributions for each fitting parameter. The range of each parameter has been chosen to include all combinations of parameters that result in acceptable fits of the top 100 best-fitting models to the data. The cross-hatched regions indicate the parameter values that produce the optimum fit to the data. 

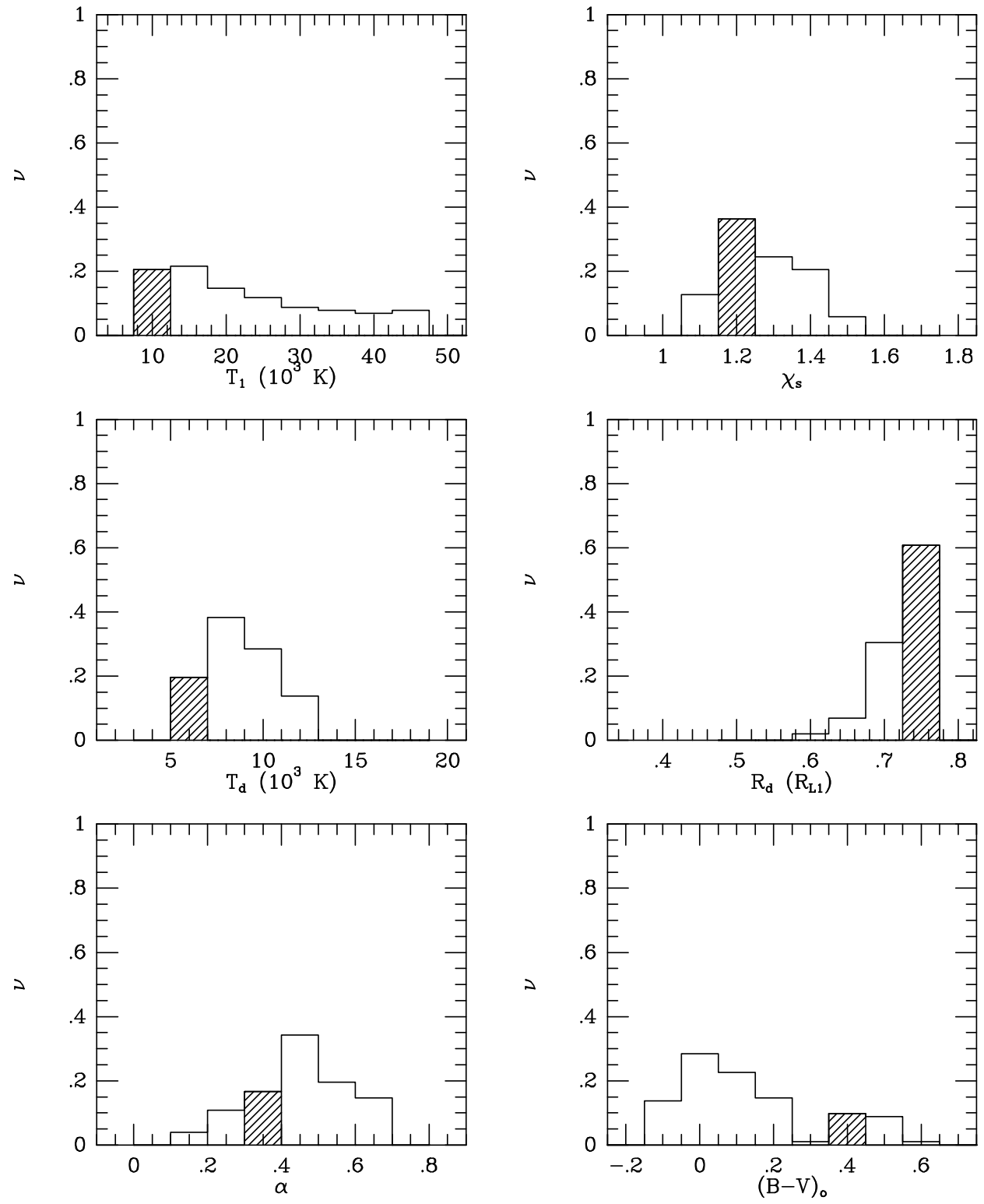

Fig. 4. - The same as Figure 3, but for $h_{\mathrm{r}}=0.04$. 

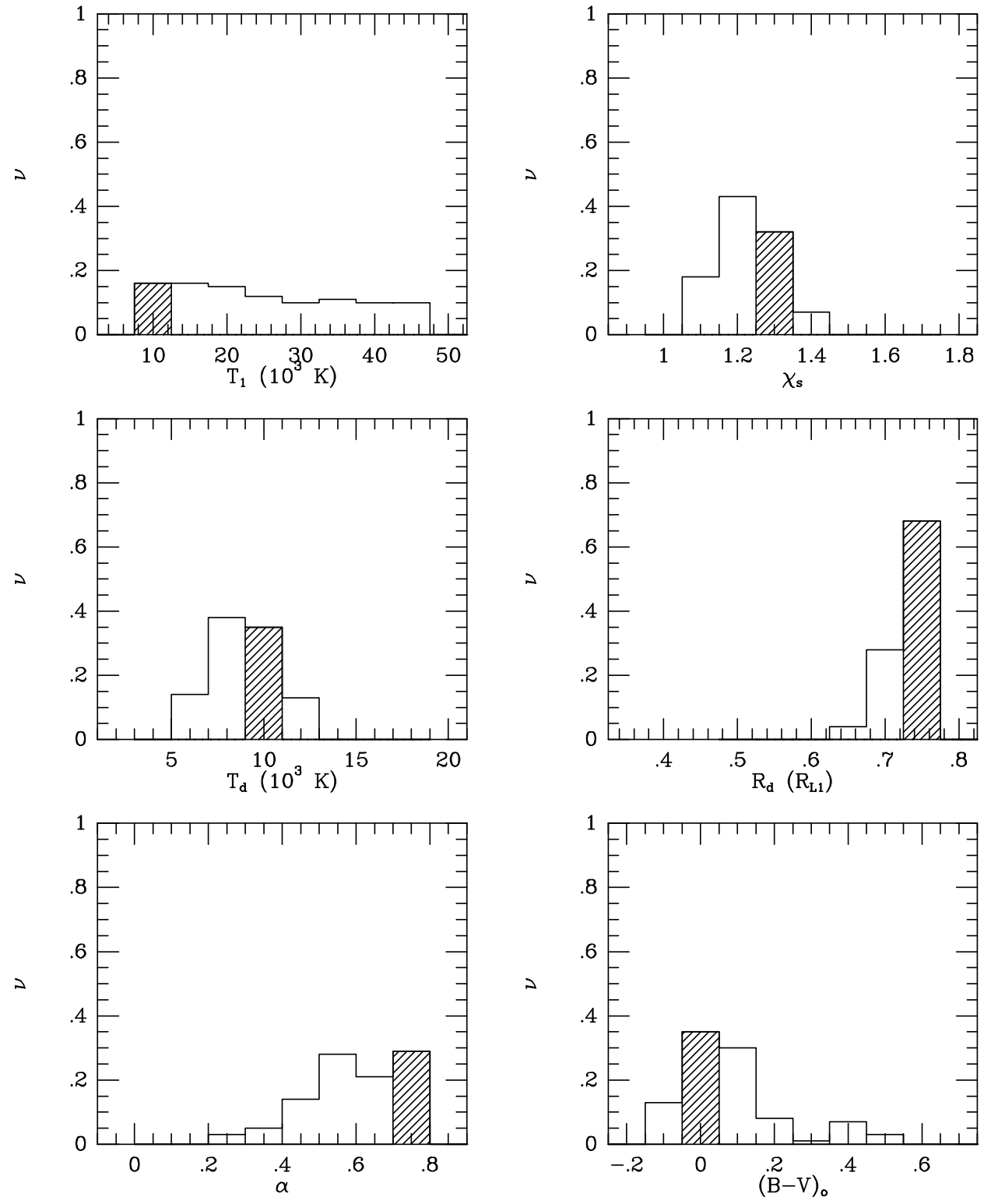

Fig. 5. - The same as Figure 3, but for $h_{\mathrm{r}}=0.08$. 


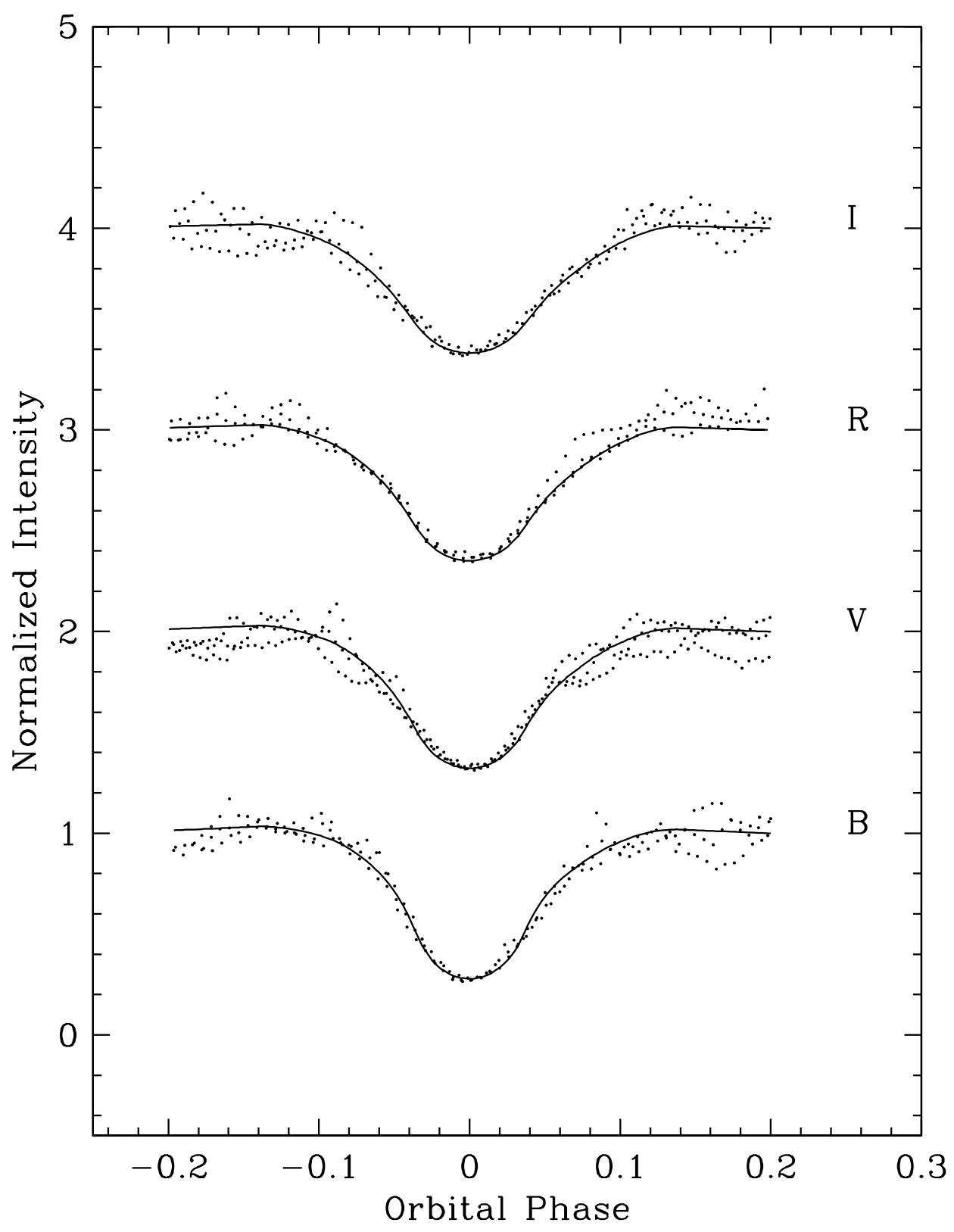

Fig. 6. - The best-fitting eclipse profiles (solid lines) for the $q=0.20, h_{\mathrm{r}}=0.08$ model are plotted together with the observed data. The data have been converted to relative intensity and normalized to unity in the orbital phase range: $0.15<\phi<0.20$. For clarity of presentation the $V, R$, and $I$-band light curves and models have been shifted upward by constant offsets of 1,2 , and 3 , respectively. 


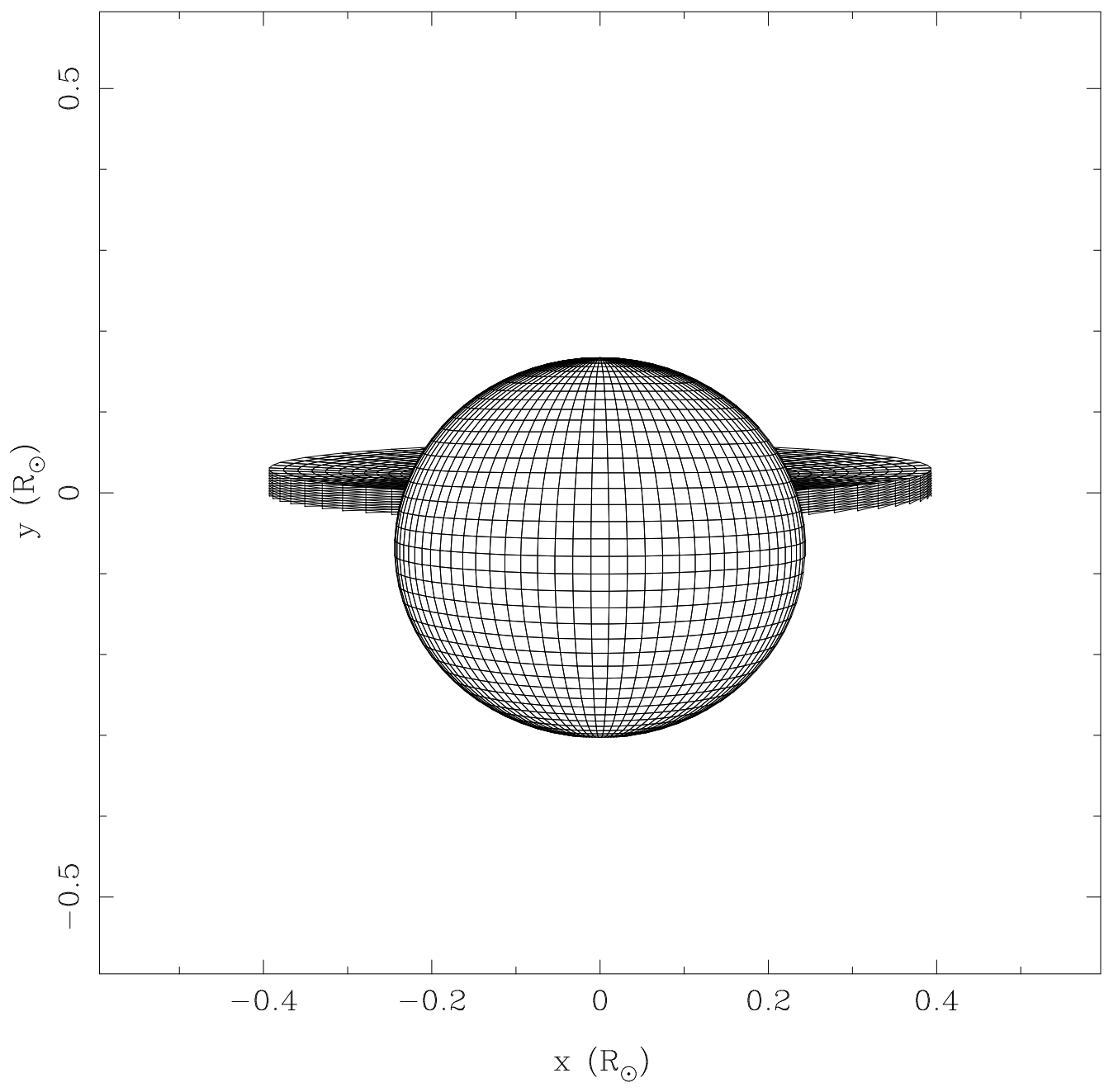

Fig. 7.- A schematic representation of the $\mathrm{V}$ Per geometry at mid-eclipse for the $q=$ $0.2, i=85.4, h_{\mathrm{r}}=0.04$ model. Note that the accretion disk is never completely eclipsed, and that the outer disk and rim make a significant contribution to the light at mid-eclipse. 


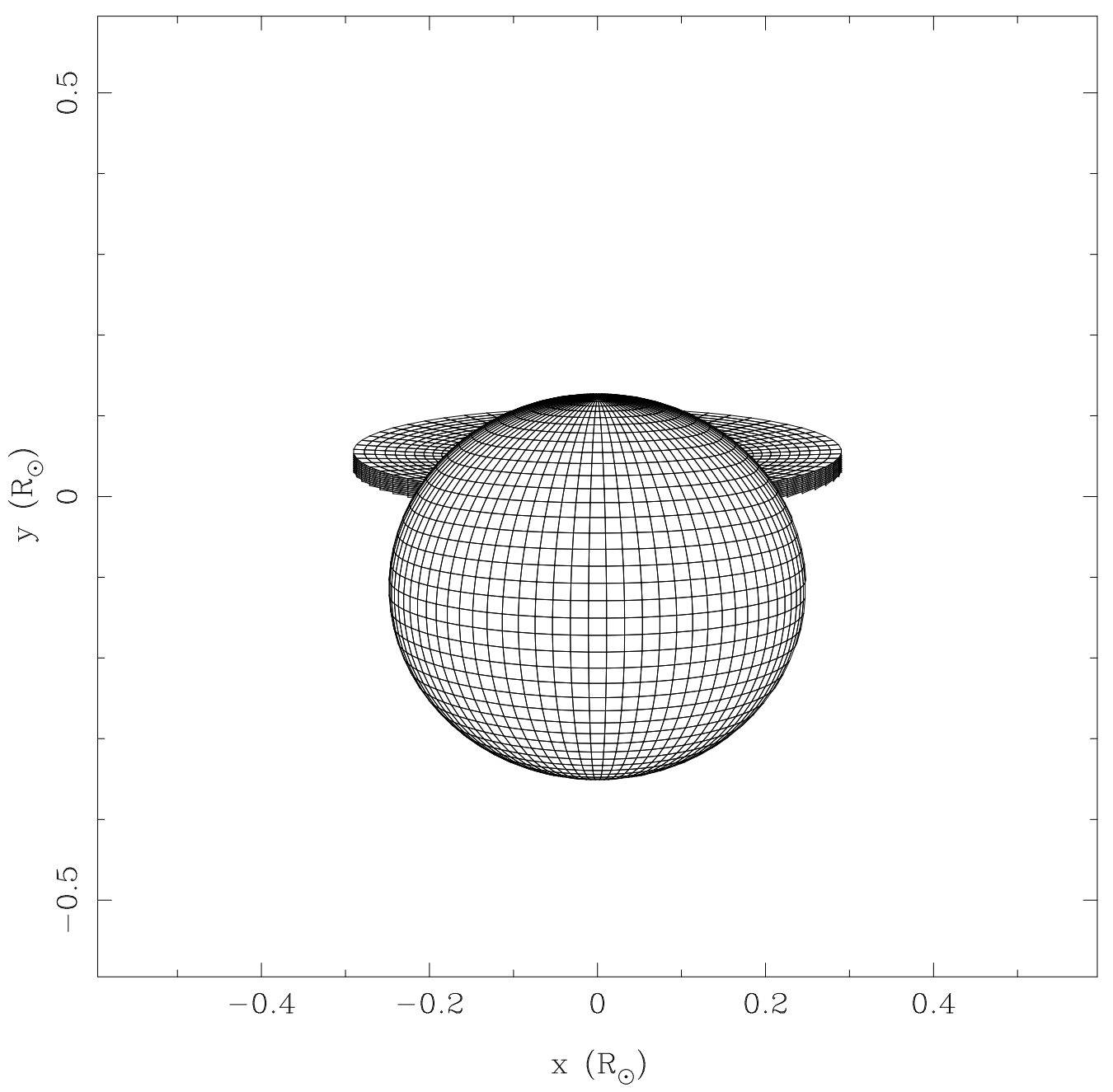

Fig. 8. - The same as Figure 7, but for $q=0.4, i=79.4, h_{\mathrm{r}}=0.04$. 

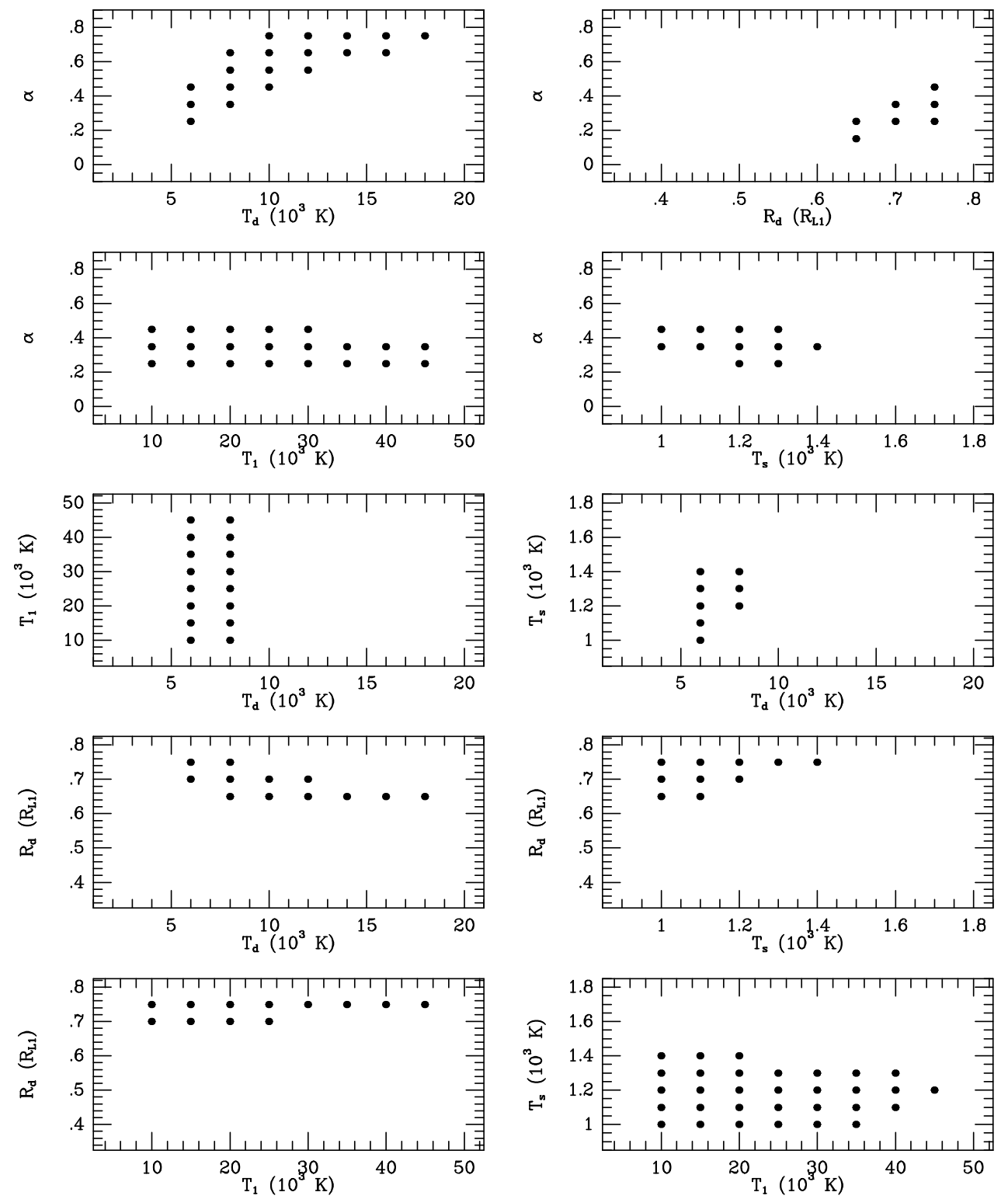

Fig. 9.- The correlations between pairs of model parameters for the representative $q=$ $0.20, h_{\mathrm{r}}=0.04$ model are shown for each of the 10 possible pairings of the five fitting parameters. In each case a range of model solutions $\left(\chi_{\nu}^{2}<2.0\right)$ for each pair of parameters is plotted, while the remaining three parameters are held fixed at their optimum values (Table 7). 